\title{
The Monodromy Rings of a Class of Self-Energy Graphs
}

\author{
G. Ponzano, T. Regge, E. R. Speer ${ }^{\star}$, and M. J. Westwater ${ }^{\star}$ \\ Institute for Advanced Study, Princeton, New Jersey
}

Received April 18, 1969

\begin{abstract}
The monodromy rings of self-energy graphs, with two vertices and an arbitrary number of connecting lines, are determined.
\end{abstract}

\section{§ 1. Introduction}

This paper is the first of a series of publications in which we hope to elucidate in a systematic way the properties of Feynman integrals. The motivation for this work is clear: we hope to develop sufficiently the methods of investigating functions of several complex variables defined by integrals to give a basis for the determination of the analytic structure of the $S$-matrix itself. This is admittedly not an easy task and one whose outcome we cannot guarantee. An ideal research program should be carried out in three steps:

I) The individual contributions of each perturbation order should be separately investigated. These are functions of the Nilsson class ${ }^{1}$ and therefore their analytic structure admits a simple qualitative description - to each function corresponds a certain group, the fundamental group of its domain, and a finite dimensional representation of this group by linear transformations of the vector space spanned by the determinations of the function in the neighbourhood of a nonsingular point. This representation may be extended to a representation of the group ring of the fundamental group which we term the monodromy ring of the function ${ }^{2}$. These rings are to be explicitly determined.

This point of the program is well under way and has been completed for the single loop Feynman relativistic amplitudes (FRA) and for the class of self-energy FRA considered in the present paper.

* Research sponsored by the Air Force Office of Scientific Research, Office of Aerospace Research, United States Air Force, under AFOSR Grant 68-1365.

${ }^{1}$ See $\S 3.3$.

2 These concepts are developed in detail in $\S 2$. 
It is important to know to what extent a function is defined by its monodromy ring. This question was posed already by Riemann who initiated the qualitative approach to the theory of functions. For functions of a single complex variable Riemann's problem has been given a complete and beautiful solution [1]:

Given a representation of the group ring of the fundamental group of the complex plane minus a finite set of points, which is finite dimensional and has a cyclic vector, there exist $n$ functions having these points as branch points such that

a) These functions have the given representation as their monodromy ring.

b) They are linearly independent over the field of rational functions.

c) Any function having the given representation as its monodromy ring is a linear combination of the $n$ basic functions with rational functions as coefficients.

We expect that this theorem remains valid also for functions of several complex variables.

II) The second step is heuristic.

The aim is to determine the simplest possible analytic structure for the $S$-matrix which would be consistent with known physical principles. For an account of the work which has been done in this direction we refer to [2]. We remark only that the unitarity condition dictates that the $S$-matrix must have a very complicated structure. The close correspondence between unitarity integrals and Feynman integrals indicates that the $S$-matrix has an analytic structure which is closely related to that of the terms in the perturbation series. We expect that the techniques developed in I) will be essential to obtain the full implication of the unitarity condition and so to give a precise statement of the analytic structure of the $S$-matrix.

III) The problem of constructing an $S$-matrix which has the structure suggested by II) must be solved. This problem can be regarded as a farreaching generalization of the problem of Riemann mentioned above.

Even in the case in which a complete solution of I) is obtained, a complete solution of II), III) would almost certainly remain out of the question. However, a partial solution - which would be in effect an extension of the original calculational scheme of Mandelstam to deal with many particle processes - would be of great interest.

We conclude this introduction with some remarks on the technical ideas introduced in this paper.

a) In contrast with previous work aimed at the determination of the monodromy rings of Feynman integrals $[3,4]$, we are able to avoid the use of homology theory. It was remarked already by Pham [4] that a 
knowledge of the fundamental group gives considerable information about the Kronecker indices which must be determined in the homological method. We have found that taken together with the information which is obtained by a purely local analysis in the integration space, the fundamental group determines completely the monodromy ring. We have no proof that this is the case for an arbitrary graph but it is not necessary to know in advance that the method will be successful in order to apply it.

b) We consider all the parameters which enter into the FRA as independent complex variables. In this way we exploit the fact that the FRA may be analytically continued in all these variables.

c) We use a device introduced by one of us (E. S.) in dealing with renormalization theory whereby the ordinary Feynman propagators $\left(p^{2}-m^{2}+i \varepsilon\right)^{-1}$ are replaced by $\left(p^{2}-m^{2}+i \varepsilon\right)^{-\lambda}, \lambda$ complex. In this way we achieve a double goal.

i) We are free of worries about divergent FRA. Renormalized FRA can be obtained by the Speer method, and their analytic structure determined.

ii) The $\lambda$ variables - we introduce a different $\lambda$ for each line - serve to label the lines. In this way we can see clearly the influence of each line in the whole structure. In particular we are able to state and prove a prove a theorem which describes the effect of cutting one line of the graph. If this theorem can be proved for an arbitrary graph it could play a key role in the construction of the monodromy rings of complicated graphs. For it would then be sufficient to construct the monodromy rings of the complete graphs on an arbitrary number of vertices. (The monodromy ring of a graph with multiple lines can be computed from the monodromy ring of the corresponding graph without multiple lines and the monodromy rings determined in the present paper.)

\subsection{The Fundamental Group - General Theorems}

For the definition of the fundamental group of a topological space we refer to any standard text on topology, for example [5]. In this section we cite a number of theorems on the fundamental group $\pi_{1}\left(\mathbb{P}^{m}-L ; B\right)$ of the complement in a projective space $\mathbb{P}^{m}$ over the field $\mathbb{C}$ of complex numbers of an algebraic variety $L$ of complex dimension $m-1$. $B$ denotes the base point for the loops defining $\pi_{1}$.

Definition 1.2.1. A line $\ell \subset \mathbb{P}^{m}$ is generic with respect to $L$ if $\ell$ intersects $L$ in a finite set of points equal in number to the degree $d$ of $L$.

Theorem 1.2.2 (Picard-Severi). $\pi_{1}\left(\mathbb{P}^{m}-L ; B\right)$ is generated by the elements $\alpha_{1}, \ldots, \alpha_{d}$ defined by elementary loops in $\ell$ around the points of intersection of a generic line $\ell$ through $B$ with L (Fig. 1). 


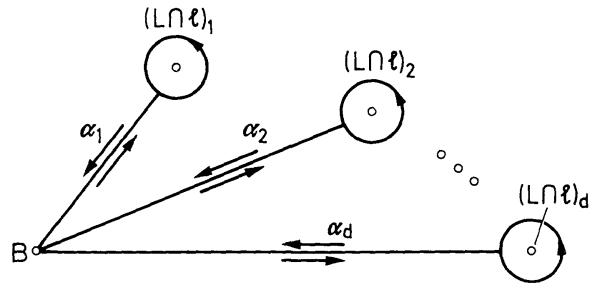

Fig. 1. The complex line $\ell$

Proof. See e.g. Pham [6].

Note that $\pi_{1}(\ell-L ; B)$ is the quotient of the free group $F_{d}$ on $\alpha_{1}, \ldots, \alpha_{d}$ by the normal subgroup generated by $\alpha_{1} \ldots \alpha_{d}$.

Definition 1.2.3 (Zariski). If $O \in \mathbb{P}^{m}-L$, and $N \cong \mathbb{P}^{m-1}$ is a linear subspace of $\mathbb{P}^{m}$ not containing $O$, the branch variety $V$ of $L$ relative to $O$ is the intersection with $N$ of the cone of singular (i.e. non-generic) lines on $O$ with respect to $L$. A plane $\pi \cong \mathbb{P}^{2} \subset \mathbb{P}^{m}$ is generic with respect to $L$ if there is some point $O \in \pi$ such that if $N$ is a linear subspace of $\mathbb{P}^{m}$ of dimension $m-1$ not containing $O$, the line $\pi \cap N$ is generic with respect to the branch variety $V$ of $L$ relative to $O$.

Theorem 1.2.4 (Zariski [7]). If $\pi$ is generic with respect to $L$ the natural injection

$$
i: \pi_{1}(\pi-L ; B) \rightarrow \pi_{1}\left(\mathbb{P}^{m}-L ; B\right)
$$

(which by 1.2.2 is onto) is an isomorphism.

Combining Zariski's theorem with the Picard-Severi theorem we see that $\pi_{1}\left(\mathbb{P}^{m}-L ; B\right)$ is a finitely generated group with generators $\alpha_{1}, \ldots, \alpha_{d}$ and that all the relations on these generators may be found by considering homotopies within a generic plane $\pi$ on $B$.

Let $\pi$ be generic for $L$. We may choose the base point $B$ to be the point $O$ of Zariski's definition and the generic line $\ell$ used to construct generators for $\pi_{1}\left(\mathbb{P}^{m}-L ; B\right)$ to be the line $B Q_{0}$, where $Q_{0} \in \pi \cap N$ is any point of $\pi \cap N$ other than the $s$ points $Q_{1} \ldots Q_{s}$ in which $\pi \cap N$ intersects the branch variety $V$ of $L$ relative to $B$. Denote by $u$ a complex variable parametrising $\pi \cap N$ so that $Q_{i}$ corresponds to $u=u_{i} 0 \leqq i \leqq s$. For $u \neq u_{1}, \ldots u_{s}$, the line $B Q(u)$ intersects $L$ in $d$ points $P_{1}(u), \ldots, P_{d}(u)$. Let $\gamma$ be a line segment in the $u$-plane connecting points $R, S$. We write $\gamma=\{\gamma(t)\}$ so $R=\gamma(0), S=\gamma(1)$. Then the motion of the points $P_{1}(\gamma(0)) \ldots P_{d}(\gamma(0))$ of intersection of $B R$ with $L$ into the points $P_{1}(\gamma(1)) \ldots P_{d}(\gamma(1))$ of $B S \cap L$ defined by continuously varying $t$ from 0 to 1 may be extended to a motion which carries an arbitrary point of the line $B R$ into a correspond- 
ing point of $B S^{3}$. The motion may be constructed so that $B$ remains fixed. It therefore defines an isomorphism

$$
\pi_{1}(B R-L ; B) \rightarrow \pi_{1}(B S-L ; B) .
$$

With the help of this construction we may assign to each piecewise linear loop $\beta$ on $Q_{0}$ in $\pi \cap N$ an automorphism

$$
h(\beta): \pi_{1}(\ell-L ; B) \rightarrow \pi_{1}(\ell-L ; B) .
$$

This automorphism depends only on the homotopy class of the loop $\beta$ in $\pi_{1}\left(\pi \cap N-V ; Q_{0}\right)$. For any $\alpha \in \pi_{1}(\ell-L ; B)$ and $\beta \in \pi_{1}\left(\pi \cap N-V ; Q_{0}\right)$ we clearly have

$$
i(\alpha)=i(h(\beta) \alpha)
$$

where $i$ denotes the injection $i: \pi_{1}(\ell-L ; B) \rightarrow \pi_{1}\left(\mathbb{P}^{m}-L ; B\right)$.

Theorem 1.2.5 (van Kampen [9]). The kernel of the injection $i$

$$
i: \pi_{1}(\ell-L ; B) \rightarrow \pi_{1}\left(\mathbb{P}^{m}-L ; B\right)
$$

is generated by the elements $\alpha[h(\beta) \alpha]^{-1}$ where

$$
\alpha \in \pi_{1}(\ell-L ; B) ; \quad \beta \in \pi_{1}\left(\pi \cap N-V ; Q_{0}\right)
$$

are arbitrary.

$\pi_{1}\left(\pi \cap N-V ; Q_{0}\right)$ is generated by elements $\beta_{1} \ldots \beta_{s}$ defined by elementary loops around $Q_{1} \ldots Q_{s}$. We may therefore combine Theorem 1.2.5 with the remark following 1.2.2 to give:

Proposition 1.2.6. $\pi_{1}\left(\mathbb{P}^{m}-L ; B\right)$ is a finitely presented group - the quotient of the free group $F_{d}$ on generators $\alpha_{1}, \ldots \alpha_{d}$ by the normal subgroup generated by

$$
\begin{aligned}
& \alpha_{1} \ldots \alpha_{d} \\
& \alpha_{i}\left[h\left(\beta_{j}\right) \alpha_{i}\right]^{-1} ; \quad 1 \leqq i \leqq d, \quad 1 \leqq j \leqq s .
\end{aligned}
$$

[In Proposition 1.2.6 $h\left(\beta_{j}\right) \alpha_{i}$ as an element of $F_{d}$ is not uniquely defined but is understood to be any element of $F_{d}$ which maps onto $h\left(\beta_{j}\right) \alpha_{i}$ considered as an element of $\pi_{1}(\ell-L ; B)$.]

We refer to the relations $\alpha_{i}\left[h\left(\beta_{j}\right) \alpha_{i}\right]^{-1}=1,1 \leqq i \leqq d$ as the van Kampen relations for the branch point. If $Q_{j}$ is the intersection with $\pi \cap N$ of the line $B P$ joining $B$ to a singular point $P$ of $L$ we may also refer to these relations as the van Kampen relations for the singular point $P$.

\footnotetext{
3 The practised reader will recognize this as an ambiant isotopy [8].
} 
Remark. The ambiant isotopy construction may be used to assign uniquely to each $\beta \in \pi_{1}\left(\pi \cap N-V ; Q_{0}\right)$ an automorphism $h^{\prime}(\beta)$ of the free group $F_{d}$ by identifying this group with $\pi_{1}(\ell-L \cup B)$. Then in 1.2 .6 we could write $\alpha_{i}\left[h^{\prime}\left(\beta_{j}\right) \alpha_{i}\right]^{-1}$. It is interesting to note that the automorphisms $h^{\prime}(\beta)$ map each generator $\alpha_{i}$ into a conjugate of some other generator $\alpha_{j}$ and map $\alpha_{1} \ldots \alpha_{d}$ into itself. They therefore define elements of the braid group $B_{d}$ on $d$ braids [10].

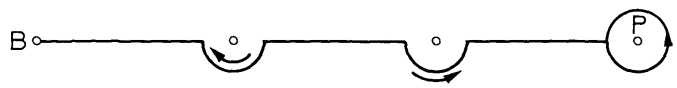

Fig. 2. The anticlockwise convention

In the application of the above results to the study of Feynman integrals we are concerned with a variety $L$ defined by a polynomial whose coefficients are real. We denote by $L_{r} \subset \mathbb{P}^{m}(R)$ the real part of $L$, $L_{r}=\mathbb{P}^{m}(R) \cap L$. As a set of real points $L_{r}$ may have components of varying topological dimension. However, the set $L_{r}-S=M$ ( $S$ the set of singular points of $L$ ), which in our case will always be nonempty is a manifold of real dimension $m-1$. We denote by $M_{i} 1 \leqq i \leqq c$ its connected components. Choose a real base point $B$. With each point $P \in M$ we associate the element $\alpha(P) \in \pi_{1}\left(\mathbb{P}^{m}-L ; B\right)$ defined by an elementary loop in the complex line $B P$ which follows the real line interval $\overline{B P}$ except for small anticlockwise detours to avoid the intersections of $B P$ with $L$ interior to $\overline{B P}$ and circles $L$ anticlockwise at $P$ (Fig. 2). Let $X$ be a connected subset of $M$. We say that $X$ is good with respect to $B$ if any two points $P, P^{\prime}$ in $X$ may be connected by a path $\gamma=\{\gamma(t)\}$ in $M$ such that as $t$ runs from 0 to 1 no complex intersection of $B P=B \gamma(0)$ crosses the interval $B \gamma(t)$. We then have $\alpha(P)=\alpha\left(P^{\prime}\right)$, i.e. we may define $\alpha(X)=\alpha(P)$ for any $P \in X$. An optimal choice of base point $B$ is clearly one such that each component $M_{i} 1 \leqq i \leqq c$ is good with respect to $B$. For the Landau variety of a single loop diagram such a base point exists [11]. For the multi-loop graphs studied in the present paper the Landau varieties do not admit such a choice of base point. However, we have been able to choose $B$ so that a large number of the components $M_{i}$ are good with respect to $B$. This choice is successful in the sense that the corresponding generators $\alpha\left(M_{i}\right)$ generate $\pi_{1}\left(\mathbb{P}^{m}-L ; B\right)$ and such that the relations on these generators obtained by writing down van Kampen relations for certain real branch points completely define the group. Note that the completeness of the set of elements $\left\{\alpha\left(M_{i}\right)\right\}$ is essential for our purpose, but that the completeness of the set of relations which we write down is not essential. It is sufficient to have sufficient relations to reconstruct uniquely the representation $\mathscr{L}$ of the fundamental group. 
A Landau variety $L$ is a reducible algebraic variety. The singular points of a generic plane section $\pi$ of $L$ are therefore expected to be of the following types

(i) transverse intersection,

(ii) tacnode,

(iii) cusp.

We are interested particularly in real singular points (since we try to avoid having to write down van Kampen relations for complex singular points). As a singular point of the real section a transverse intersection or node can appear either as a transverse intersection of two branches of $L_{r}$ (crunode) or as an isolated realpoint (acnode). Since we wish to consider only van Kampen relations which can be written down as relations on elements of the fundamental group defined by elementary loops around points of $L_{r}$ we do not consider the acnode case. This gives us the three cases illustrated in Figs. 3, 4, 5. In the neighbourhood of the singular point $P$ local coordinates $u_{1}, u_{2}$ may be chosen so that $L$ has local equation

(i) $\left(u_{1}-u_{2}\right)\left(u_{1}+u_{2}\right)=0$,

(ii) $\left(u_{1}^{2}-u_{2}\right) u_{2}=0$

(iii) $u_{2}^{2}-u_{1}^{3}=0$.

We choose a base point $B$ such that $P$ is the only singular point of $L$ on $B P$ and such that $B P$ does not touch $L$, i.e. we choose $B$ to be a point $O$ satisfying the conditions of 1.2.3. We also choose $B$ so that in cases (ii), (iii) $B$ stands in the relative position to the real section of $L$ in the neighbourhood of $P$ indicated in Figs. 4 and 5. Then if $U$ is a sufficiently small neighbourhood of $P$ the connected components $K_{i}$ of $U \cap\left(L_{r}-P\right)$ are good with respect to $B$. We denote by $\alpha_{i}=\alpha\left(K_{i}\right)$ the corresponding elements of the fundamental group constructed by the anticlockwise convention, the labelling being carried out as shown in the figures. We will work out in detail the relations between these generators for the tacnode. Choose as the generic line $\ell$ the line $B P^{\prime}$ for some $P^{\prime} \in K_{1}$, and let $\pi \cap N$ be a line defined as in the general discussion preceding the statement of the van Kampen theorem (Fig. 6). Let $\pi \cap N$ intersect $B P$, $B P^{\prime}=\ell$ in $Q, Q_{0}$. The construction of the van Kampen theorem now gives us two kinds of relations

(a) identifications obtained by taking for the path $\gamma$ in $\pi \cap N$ a path from $Q_{0}=R$ to a point $S$ on the opposite side of $Q$ circling $Q$ anticlockwise. By following the motion of the intersections of $B \gamma(t)$ with $L$ as $t$ traces this path we can express the generators defined by $B S$ in terms of those defined by $\ell$. For the remaining $d-2$ generators this results in trivial identifications but we do obtain two non-trivial relations expressing $\alpha_{3}, \alpha_{4}$ in terms of $\alpha_{1}, \alpha_{2}$; 


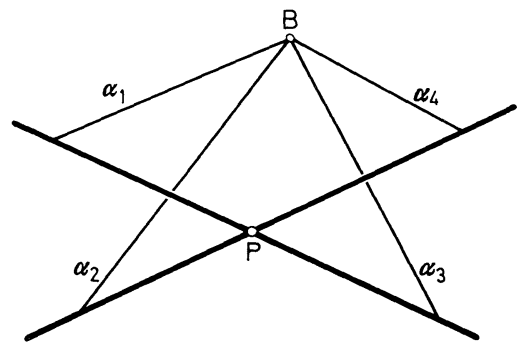

Fig. 3. Crunode

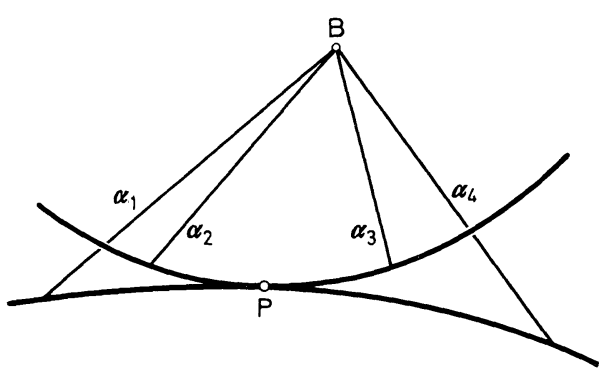

Fig. 4. Tacnode ${ }^{\star}$

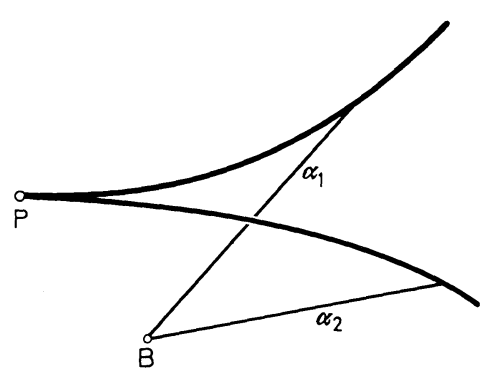

Fig. 5. Cusp

(b) the van Kampen relations for $P$ obtained by taking a loop $\beta$ around $Q$ in $\pi \cap N$.

The task of following the motion of the loops in $B \gamma(t)$ obtained by deformation of $\alpha_{1}, \alpha_{2}$ may be reduced to successive applications of

$\star$ The labels $\alpha_{1}$ and $\alpha_{2}$ in Fig. 4 should be interchanged. 


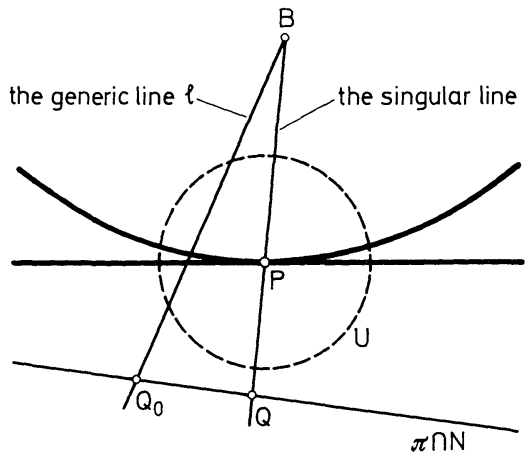

Fig. 6. The neighbourhood of a tacnode

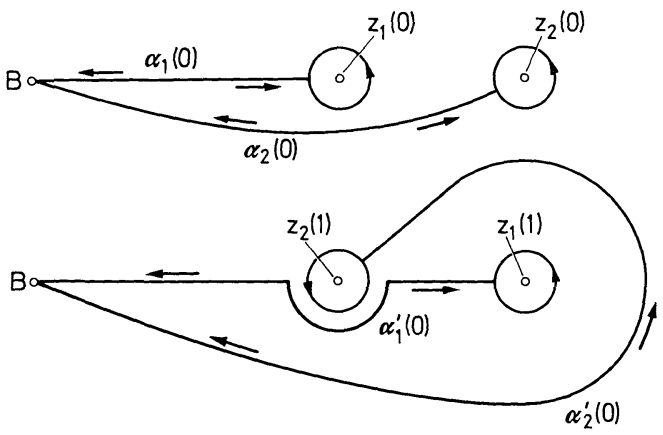

Fig. 7. z-plane

Lemma 1.2.7 (Fig. 7). Let $z_{1}(u), z_{2}(u)$ be two points of the complex $z$-plane depending continuously on a parameter $u$. Suppose that for $u=1,0$ the points are real and satisfy

$$
z_{1}(0)<z_{2}(0), \quad z_{2}(1)<z_{1}(1)
$$

Suppose also that $\operatorname{Im}\left(z_{2}(u)-z_{1}(u)\right)$ is positive for any $u, 0<u<1$ (so that the points circle just once). Choose a base point $B$ far away on the negative real axis and denote by $\alpha_{1}(0), \alpha_{2}(0) ; \alpha_{1}(1), \alpha_{2}(1)$ elements of $\pi_{1}\left(\mathbb{C}^{2}-\left\{z_{1}\right\}\right.$ $\left.\cup\left\{z_{2}\right\}\right)$ defined by loops around $z_{1}, z_{2}$ constructed according to the anticlockwise convention. Then as $u$ varies from 0 to $1 \alpha_{1}(0), \alpha_{2}(0)$ are carried into

$$
\begin{aligned}
& \alpha_{1}^{\prime}(0)=\alpha_{1}(1) \\
& \alpha_{2}^{\prime}(0)=\alpha_{1}^{-1}(1) \alpha_{2}(1) \alpha_{1}(1) .
\end{aligned}
$$




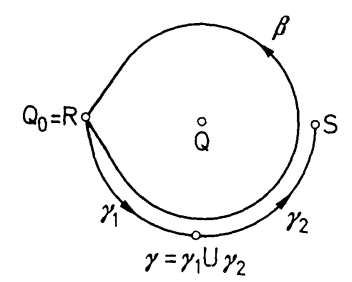

Fig. 8. The complex line $\pi \cap N$

Proof. (1.2.8) is evident. For (1.2.9) we note that the loop $\alpha_{1}(0) \alpha_{2}(0)$ is mapped into the loop $\alpha_{2}(1) \alpha_{1}(1)$, i.e.

or

$$
\alpha_{1}^{\prime}(0) \alpha_{2}^{\prime}(0)=\alpha_{2}(1) \alpha_{1}(1)
$$

in view of (1.2.9).

$$
\alpha_{2}^{\prime}(0)=\alpha_{1}^{-1}(1) \alpha_{2}(1) \alpha_{1}(1)
$$

To obtain the identification relations for the tacnode we write the path $\gamma$ as the union of paths $\gamma_{1}, \gamma_{2}$, arcs circling one quarter the way round $Q$ (Fig. 8). Lemma 1.2.7 gives the relations between the generators in the lines $B \gamma_{1}(0), B \gamma_{1}(1)$ and between those on $B \gamma_{2}(0)=B \gamma_{1}(1)$ and $\mathrm{B} \gamma_{2}(1)$. Hence we obtain

$$
\begin{aligned}
& \alpha_{1}=\alpha_{4}^{-1} \alpha_{3} \alpha_{4}, \\
& \alpha_{2}=\left(\alpha_{3} \alpha_{4}\right)^{-1} \alpha_{4}\left(\alpha_{3} \alpha_{4}\right) .
\end{aligned}
$$

To obtain the van Kampen relations we write down the identification relations obtained by linking $S$ to $R$ by the semicircular arc $\beta-\gamma$

$$
\begin{array}{r}
\alpha_{3}=\alpha_{2}^{-1} \alpha_{1} \alpha_{2}, \\
\alpha_{4}=\left(\alpha_{1} \alpha_{2}\right)^{-1} \alpha_{2}\left(\alpha_{1} \alpha_{2}\right),
\end{array}
$$

and eliminate $\alpha_{3}, \alpha_{4}$ to give

$$
\begin{aligned}
& \alpha_{1}=\left(\alpha_{1} \alpha_{2}\right)^{-1} \alpha_{2}^{-1}\left(\alpha_{1} \alpha_{2}\right)\left(\alpha_{2}^{-1} \alpha_{1} \alpha_{2}\right)\left(\alpha_{1} \alpha_{2}\right)^{-1} \alpha_{2}\left(\alpha_{1} \alpha_{2}\right), \\
& \alpha_{2}=\left(\alpha_{1} \alpha_{2}\right)^{-2} \alpha_{2}\left(\alpha_{1} \alpha_{2}\right)^{2} .
\end{aligned}
$$

The first of these relations is a consequence of the second which may be written

$$
\left(\alpha_{1} \alpha_{2}\right)^{2}=\left(\alpha_{2} \alpha_{1}\right)^{2} \text {. }
$$

In view of (1.2.14), (1.2.13) simplifies to

$$
\alpha_{4}=\alpha_{1} \alpha_{2} \alpha_{1}^{-1} \text {. }
$$

If two elements $\alpha_{1}, \alpha_{2}$ of a group satisfy (1.2.14) we say that they $b i$ commute and write $\alpha_{1} \uplus \alpha_{2}$.

The relations between generators in the crunode and cusp cases are worked out in the same way (again using Lemma 1.2.7). The results are 
Table. Relations between group elements in the neighbourhood of a singular point

\begin{tabular}{|c|c|c|}
\hline Singularity type & Identification relations & van Kampen relation \\
\hline $\begin{array}{l}\text { Crunode (Fig. 3) } \\
\text { Tacnode (Fig. 4) } \\
\text { Cusp (Fig. 5) }\end{array}$ & $\begin{array}{ll}\alpha_{1}=\alpha_{3} & \alpha_{2}=\alpha_{4} \\
\alpha_{3}=\alpha_{2}^{-1} \alpha_{1} \alpha_{2} & \alpha_{4}=\alpha_{1} \alpha_{2} \alpha_{1}^{-1}\end{array}$ & $\begin{array}{l}\alpha_{1} \alpha_{2}=\alpha_{2} \alpha_{1} \\
\left(\alpha_{1} \alpha_{2}\right)^{2}=\left(\alpha_{2} \alpha_{1}\right)^{2} \\
\alpha_{1} \alpha_{2} \alpha_{1}=\alpha_{2} \alpha_{1} \alpha_{2}\end{array}$ \\
\hline
\end{tabular}

given in Table 1 . Note that in the cuspidal case $\alpha_{3}, \alpha_{4}$ are elements defined by loops around complex points of $L$ so we do not write down the identification relations in this case.

\section{$\S 2$. Determination of the Monodromy Rings in the Generic Case}

2.1. The Feynman Integrals Associated with the Graphs $G_{N}$ (Fig. 9)

In a space-time of dimension $m$ the Feynman integral associated with $G_{N}$ in a theory in which all particles have spin 0 is given as a function of the energy $s_{0}$ and the masses $s_{i} 1 \leqq i \leqq N$ of the exchanged particles by the integral

$$
I(s)=\int \prod_{i=1}^{N} \frac{d^{m} k_{i}}{k_{i}^{2}+s_{i}} \delta\left(\Sigma k_{i}-p\right), \quad\left(p^{2}=s_{0}\right) .
$$

(2.1.0) can be written in the parametric form

$$
I(s)=\Gamma\left(-\frac{m}{2}(N-1)+N\right) \int_{\Delta} \frac{\delta\left(\sum \alpha_{i}-1\right) d \alpha_{1} \ldots d \alpha_{N}}{[d(\alpha)]^{m / 2}[D / d]^{-(m / 2)(N-1)+N}} .
$$

The integration region $\Delta$ is the simplex

$$
\Delta=\left\{(\alpha): \alpha_{i} \geqq 0 \forall i, \sum_{i=1}^{N} \alpha_{i}=1\right\} ;
$$

$d(\alpha), D(s, \alpha)$ are the Symanzik functions

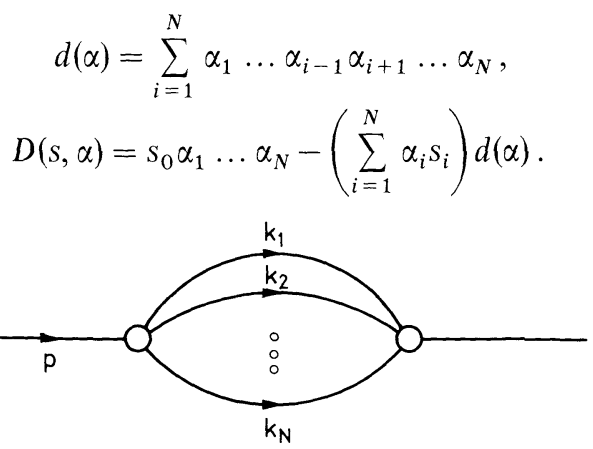

Fig. 9. The self-energy graph $G_{N}$ 
The integral (2.1.0) is convergent only in the cases

$$
\begin{array}{ll}
m=2, & N \text { arbitrary }, \\
m=3, & N=2 .
\end{array}
$$

For the remaining values of $m(\geqq 2)$ and $N$, the integral (2.1.0) diverges and we must study the renormalized integral defined by a suitable renormalization procedure (see $\S 4$ ).

(2.1.1) may be rewritten in a form in which the integrand is more symmetric. Define

$$
\begin{aligned}
\alpha_{0} & =-\frac{\alpha_{1} \ldots \alpha_{N}}{d(\alpha)}, \\
g(\alpha) & =\sum_{i=0}^{N} \alpha_{i}^{-1}, \\
D^{\prime}(s, \alpha) & =\sum_{i=0}^{N} s_{i} \alpha_{i} .
\end{aligned}
$$

Then up to a sign

$$
\begin{aligned}
I(s) & =\Gamma\left(-\frac{m}{2}(N-1)+N\right) \\
& \cdot \int_{\Delta^{\prime}} \frac{\delta\left(\sum_{i=1}^{N} \alpha_{i}-1\right) \delta(g(\alpha)) \alpha_{0}^{(m / 2)-2} \alpha_{1}^{-m / 2} \ldots \alpha_{N}^{-m / 2} d \alpha_{0} \ldots d \alpha_{N}}{\left[D^{\prime}(\alpha)\right]^{-(m / 2)(N-1)+N}},
\end{aligned}
$$

where

$$
\Delta^{\prime}=\left\{(\alpha): \alpha_{i} \geqq 0 \quad 1 \leqq i \leqq N, \sum_{i=1}^{N} \alpha_{i}=1, g(\alpha)=0\right\} .
$$

The integrand in (2.1.10) has the form

$$
\delta\left(\sum_{i=1}^{N} \alpha_{i}-1\right) f(\alpha) d \alpha_{0} \ldots d \alpha_{N},
$$

where $f(\alpha)$ is homogeneous in $\alpha$ of degree

$$
1+\left(\frac{m}{2}-2\right)-\frac{m}{2} N+\frac{m}{2}(N-1)-N=-(N+1)
$$

so (2.1.10) can be rewritten as an integral in projective space [12] (again up to a sign)

$$
I(s)=\Gamma\left(-\frac{m}{2}(N-1)+N\right) \int_{A^{\prime \prime}} \frac{\delta(g(\alpha)) \alpha_{0}^{(m / 2)-2} \alpha_{1}^{-m / 2} \ldots \alpha_{N}^{-m / 2} \eta}{\left[D^{\prime}(\alpha)\right]^{-(m / 2)(N-1)+N}} .
$$


$\eta$ is the fundamental projective form

and

$$
\begin{gathered}
\eta=\sum_{i=0}^{N}(-1)^{i} \alpha_{i} d \alpha_{0} \ldots d \alpha_{i-1} d \alpha_{i+1} \ldots d \alpha_{N} \\
\Delta^{\prime \prime}=\left\{(\alpha): \alpha_{i} \geqq 0 \quad 1 \leqq i \leqq N\right\} \subset \mathbb{P}^{N} .
\end{gathered}
$$

In order to study the monodromy ring of $I(s)$ we choose a reference point $B=(1, \varepsilon, \ldots, \varepsilon)$ in the space

$$
\mathbb{C}^{N+1}=\left\{(s)=\left(s_{0}, s_{1}, \ldots, s_{N}\right)\right\} .
$$

Here $\varepsilon$ is a positive number, sufficiently small so that $B$ lies above the normal threshold $\sqrt{s_{0}}=\sqrt{s_{1}}+\cdots+\sqrt{s_{N}}$, i.e.

$$
N^{2} \varepsilon<1 \text {. }
$$

(2.1.12) is not an integral of standard form but it may be shown that the ambiant isotopy component of $B$ for a suitable resolution of (2.1.12) into standard form is the complement in $\mathbb{C}^{N+1}$ of the set

where

$$
L=\bigcup_{i=0}^{N+1} L_{i}
$$

$$
\begin{aligned}
L_{i} & \left.=\{(s)\}: s_{i}=0\right\} \quad 0 \leqq i \leqq N \\
L_{N+1} & =\left\{(s): \pm \sqrt{s_{0}} \pm \sqrt{s_{1}} \pm \cdots \pm \sqrt{s_{N}}=0\right\}
\end{aligned}
$$

Define $\mathscr{G}_{N}$ to be the fundamental group

$$
\mathscr{G}_{N}=\pi_{1}\left(\mathbb{C}^{N+1}-L ; B\right)
$$

and $V_{N}$ to be the vector space spanned by germs of $I(s)$ with centre $B$. Then we have a representation ${ }^{4}$

$$
\mathscr{L}: \mathscr{G}_{N} \rightarrow L\left(V_{N}\right)=G L(d, \mathbb{C}) \quad d=\operatorname{dim} V_{N}
$$

defined by assigning to each loop on $B$ the linear transformation of $V_{N}$ induced by analytic continuation along this loop. $\mathscr{L}$ can evidently be extended to a representation of the group ring $\mathbb{C}\left(\mathscr{G}_{N}\right)$ of $\mathscr{G}_{N}$ over the complex field $\mathbb{C}$ into $L\left(V_{N}\right)$. We wish to study the monodromy ring

$$
\mathscr{A}_{N}=\mathscr{L}\left(\mathbb{C}\left(\mathscr{G}_{N}\right)\right) \subset L\left(V_{N}\right) \text {. }
$$

It is important to note that the ambiant isotopy component of $B$ is completely determined by the location of the singularities of the integrand

\footnotetext{
${ }^{4}$ The fact that $d$ is finite is established in the course of our investigation.
} 
for $I(s)$. It is thus natural to introduce the more general integral

$$
I(s, \lambda)=\Gamma\left(\Sigma \lambda_{i}+(N+1)\right) \int_{\Delta^{\prime \prime}} \frac{\delta(g(\alpha)) \prod_{i=0}^{N} \alpha_{i}^{\lambda_{2}}}{\left[D^{\prime}(\alpha)\right]^{\Sigma \lambda_{1}+(N+2)}} \eta,
$$

which again defines an analytic function on $\mathbb{C}^{N+1}-L$. The exponent of $D^{\prime}(\alpha)$ is determined by the requirement that the integrand be a projective form. We obtain for arbitrary complex $\lambda_{i}$, such that (2.1.18) is convergent, a representation $\mathscr{L}(\lambda)$ of the same group $\mathscr{G}_{N}$ and a monodromy ring

$$
\mathscr{A}_{N}(\lambda)=\mathscr{L}(\lambda)\left(\mathbb{C}\left(\mathscr{G}_{N}\right)\right)
$$

We refer to the ring $\mathscr{A}_{N}(\lambda)$ associated with $G_{N}$ for generic $\lambda_{i}$ (i.e. $\lambda_{i}$ not satisfying certain equations - in particular non-integral $\lambda_{i}$ ) as the generic monodromy ring for $G_{N}$. It turns out to have a very simple structure it is a complete matrix ring over a vector space of dimension $2^{N}-1$ (cf. § 2.4). Nevertheless the ring $\mathscr{A}_{N, m=2}$ of the original integral with $m=2$ can be obtained from $\mathscr{A}_{N}(\lambda)$ by specializing the $\lambda_{i}$ to the values $\lambda_{i}^{0}$ appearing in (2.1.12). For $I(s, \lambda)$, regarded as a function defined on the universal covering space of $\mathbb{C}^{N+1}-L$, is continuous in $\lambda$ in the nhd. of $\lambda_{i}^{0}$, uniformly for $s$ in any compact set. The specialization is carried out in $\S 3$.

\subsection{The Fundamental Group $\mathscr{G}_{N}$}

We construct a set of elements which generate $\mathscr{G}_{N}$ by choosing certain representative loops on $B$ in the space $\mathbb{C}^{N+1}-L . \mathscr{G}_{N}$ is the quotient of the free group on these generators by the normal subgroup defined by the van Kampen relations for singular points of $L$, nodes or tacnodes, lying in or on the boundary of the region

$$
\left\{(s): s_{i} \geqq 0 \forall i\right\} .
$$

We will need to write down explicitly only certain of these van Kampen relations, since these turn out to give sufficient information to construct the monodromy ring $\mathscr{A}_{N}(\lambda)$.

In constructing loops to define elements of $\mathscr{G}_{N}$ we follow the anticlockwise convention of $\S 1.2$. (Fig. 2). The following components of $L_{r}-S$ are good with respect to the base point $B$ chosen in $\S 2.1$. (This is strictly true only in the limit $\varepsilon \rightarrow 0$. For small finite $\varepsilon$ a set of points whose relative measure $\rightarrow 0$ as $\varepsilon \rightarrow 0$ must be deleted from the components. However, this does not affect the presentation we obtain for $\mathscr{G}_{N}$.)

$$
\begin{aligned}
& L_{i}^{0)}=\left\{(s): s_{i}=0 \quad s_{j}>0 j \neq i \sqrt{s_{0}}>\sum_{j \neq 0} \sqrt{s_{j}}\right\} . \\
& L_{0}^{i)}=\left\{(s): s_{0}=0 \quad s_{j}>0 j \neq 0 \sqrt{s_{i}}>\sum_{j \neq i} \sqrt{s_{j}}\right\} .
\end{aligned}
$$




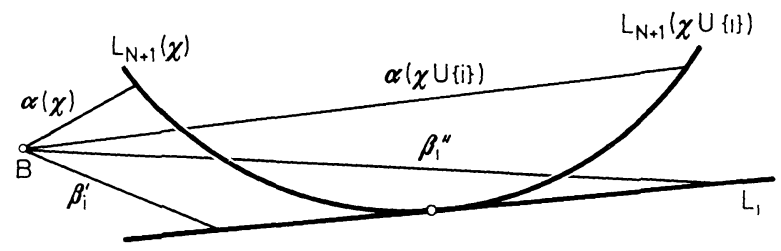

Fig. 10. Tacnodal contact of $L_{2}$ and $L_{N+1}$

We write $\alpha\left(L_{i}^{0}\right)=\beta_{i} 1 \leqq i \leqq N, \alpha\left(L_{0}^{i)}\right)=\beta_{0}^{i)} 1 \leqq i \leqq N$. Also for any proper subset $\chi$ of $\Omega=\{1, \ldots, N\}$ any component of $L_{r}-S$ contained in

$$
L_{N+1}(\chi)=\left\{(s): s_{i}>0 \quad \text { for all } \dot{i} \quad \sqrt{s_{0}}+\sum_{i \in \chi} \sqrt{s_{i}}=\sum_{i \notin \chi} \sqrt{s_{i}}\right\} .
$$

These components are separated only by transverse intersections so we may make immediate use of the identification relations for a transverse intersection (Fig. 3) and introduce one element $\alpha(\chi)$ of $\mathscr{G}$ defined by any one of them.

Any real nonsingular point of $L$ in the component $L_{i}$ for some $i, 1 \leqq i \leqq N$, can be joined in $L_{i}$ to a point in $L_{i}^{0)}$ by a real path which intersects the set of singular points of $L$ only in nodal points or in tacnodal points of the kind shown in Fig. 10. [In Fig. 10 the loop corresponding to a point $P$ of $L_{r}$ is indicated by the line $B P$.] The identification relations for a tacnode are simplified by the position of the base point $B$ ( $B$ is not in the symmetrical position considered in $\S 1.2)$ - the elements $\beta_{i}^{\prime}, \beta_{i}^{\prime \prime}$ of $\mathscr{G}_{N}$ defined by points of $L_{i}$ on either side of the tacnode are the same. Any real nonsingular point of $L$ in $L_{i}$ thus defines the same element of $\mathscr{G}_{N}$ as a point in $L_{i}^{0)}: \beta_{i}^{\prime}=\beta_{i}^{\prime \prime}=\beta_{i}$. The remaining identification relation obtained from the tacnode shown in Fig. 10 is

$$
\alpha(\chi \cup\{i\})=\beta_{i} \alpha(\chi) \beta_{i}^{-1}
$$

and the van Kampen relation is

$$
\left(\beta_{i} \alpha(\chi)\right)^{2}=\left(\alpha(\chi) \beta_{i}\right)^{2} .
$$

Since the components $L_{i}$ of $L$ intersect transversely $1 \leqq i \leqq N$

$$
\beta_{i} \beta_{j}=\beta_{j} \beta_{i} \quad 1 \leqq i, j \leqq N
$$

Similarly we have

$$
\beta_{i} \beta_{0}^{j)}=\beta_{0}^{j)} \beta_{i} \quad i \neq j, 1 \leqq i, j \leqq N
$$

(but $\beta_{i}, \beta_{0}^{i)}$ do not commute since $L_{i}$ does not intersect the region $L_{0}^{i)}$ used to define $\beta_{0}^{i)}$ ). 


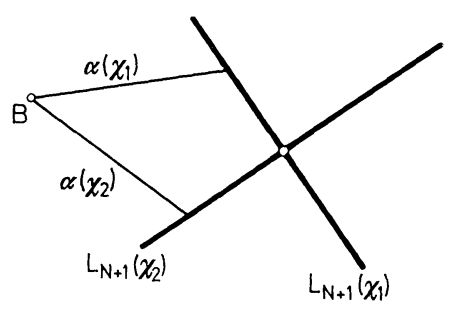

Fig. 11. Self-intersection of $L_{N+1}$

Next we consider the real self-intersections of $L_{N+1}$ in the interior of the region $\left\{(s): s_{i}>00 \leqq i \leqq N\right\}$ (Fig. 11). These are nodal points. We have already used the corresponding identification relations to define the elements $\alpha(\chi)$. We now write down the van Kampen relations. The intersection

$$
\begin{aligned}
& L_{N+1}\left(\chi_{1}\right) \cap L_{N+1}\left(\chi_{2}\right)=\left\{(s): s_{l}>0 \quad \text { for all } i\right. \\
& \left.\sqrt{s_{0}}+\sum_{i \in \chi_{1} \cap \chi_{2}} \sqrt{s_{i}}=\sum_{i \in \chi_{1}^{\prime} \cap \chi_{2}^{\prime}} \sqrt{s_{i}}, \sum_{i \in \chi_{1} \cap \chi_{2}^{\prime}} \sqrt{s_{i}}=\sum_{i \in \chi_{1}^{\prime} \cap \chi_{2}} \sqrt{s_{i}}\right\}
\end{aligned}
$$

$\left(\chi^{\prime}=\Omega-\chi\right)$ is non-empty unless $\chi_{1} \subset \chi_{2}$ or $\chi_{2} \subset \chi_{1}$ or $\chi_{1} \cup \chi_{2}=\Omega$. Thus we obtain the commutation relations

$$
\left.\begin{array}{l}
\alpha\left(\chi_{1}\right) \alpha\left(\chi_{2}\right)=\alpha\left(\chi_{2}\right) \alpha\left(\chi_{1}\right) \\
\text { for all proper subsets } \chi_{1}, \chi_{2} \text { of } \Omega \text { such that } \\
\chi_{1} \varangle \chi_{2}, \chi_{2} \varangle \chi_{1}, \chi_{1} \cup \chi_{2} \neq \Omega .
\end{array}\right\}
$$

Note that we have not named the elements of $\mathscr{G}_{N}$ for regions of $L_{0}$ other than the $L_{0}^{i)}(i=1 \ldots N)$. Neither have we written down the van Kampen relations for tacnodes on $L_{0}$. We will show below that the elements $\alpha(\chi), \beta_{i}$, and $\beta_{0}^{i}$ generate $\mathscr{G}_{N}$. The additional van Kampen relations suffice to determine $\mathscr{G}_{N}$, but will not be required for the determination of $\mathscr{A}_{N}$.

Now let $Q^{(i)}$ be the point

$$
Q^{(i)}=\left(\eta_{0}, \eta_{1} \ldots \eta_{i-1}, 1, \eta_{i+1} \ldots \eta_{N}\right),
$$

where the $\eta_{j}$ are positive and less than $\varepsilon$, and consider the (complex) line $\ell_{i}$ given by

$$
s=(1-z) B+z Q^{(i)} .
$$

This line intersects $L_{i}^{0)}$ at a real negative value $z_{i}$. It intersects $L(\chi)$, for $i \notin \chi$, at a real positive value $z(\chi)$; moreover, $z\left(\chi_{1}\right)<z\left(\chi_{2}\right)$ if $\chi_{1} \subset \chi_{2}$. Finally, it intersects $L_{0}^{i)}$ and $L_{j}(j=1, \ldots \hat{i} \ldots N)$ at real values $z_{0}, z_{j}$ which are greater than any $z(\chi)$. The situation is illustrated in Fig. 12. 


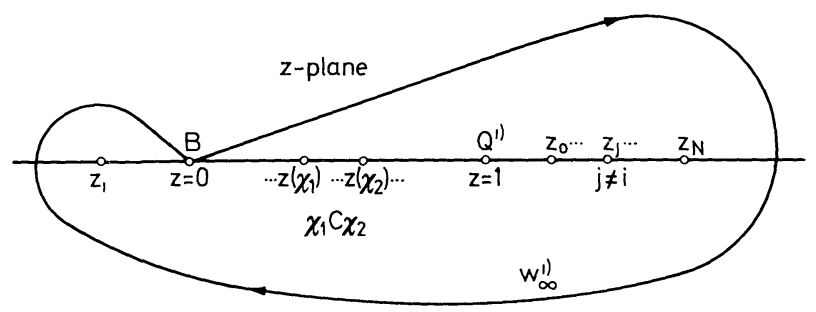

Fig. 12. The word at infinity in $\ell_{\iota}$

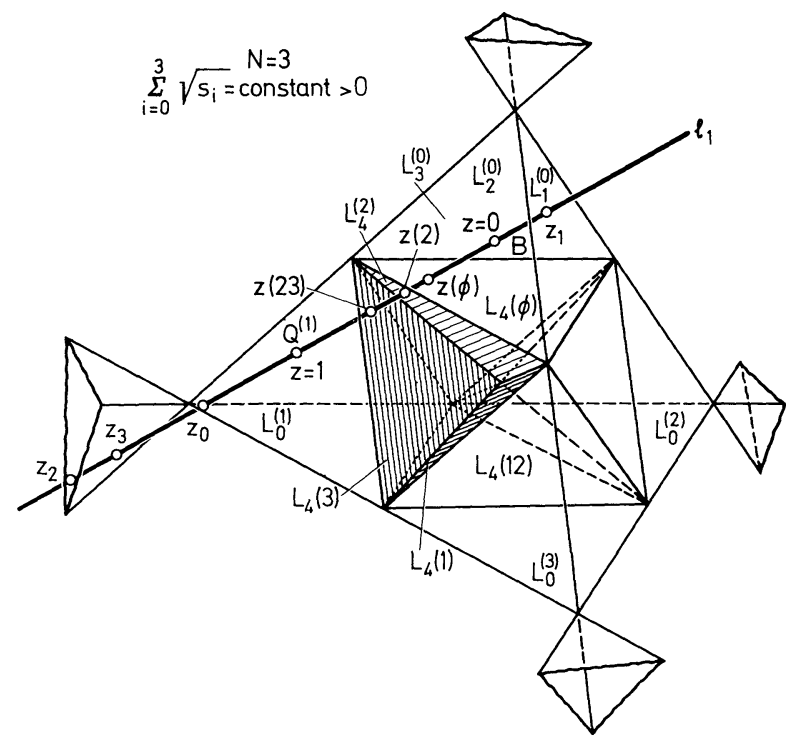

Fig. 13

Since each $L,(j=0 \ldots N)$ is of degree one in $s$, and since $L_{N+1}$ is of degree $2^{N-1}$, these real intersections are all intersections of $\ell_{i}$ with $L$. Therefore, by the Picard-Severi Theorem 1.2.2 the corresponding elements generate $\mathscr{G}_{N}$. For future reference we write down the word at infinity in $\ell_{i}$, i.e., the anticlockwise loop around the point at infinity in $\ell_{i}$ (such a loop is shown in Fig. 12):

$$
w_{\infty}^{l)^{-1}}=\beta_{i} \alpha(\phi) \prod_{j \neq i} \alpha(\{j\}) \ldots \alpha\left(\Omega_{i}\right) \beta_{0}^{i} \prod_{j \neq i} \beta_{j},
$$

where $\Omega_{i}=\{1 \ldots \hat{i} \ldots N\}$. Note that the order of elements $\alpha(\chi)$ in $(2.2 .10)$ is sufficiently determined by the order relations on the $z(\chi)$ noted above, in view of the commutation relations (2.2.9). 
We close this section by defining a homomorphism

$$
j: \mathscr{G}_{N-1} \rightarrow \mathscr{G}_{N}
$$

which will be needed in the isomorphism theorem of $\S 2.4$. The parameter space for the graph $G_{N-1}$ may be identified with the subspace $L_{N}$ of the parameter space $\mathbb{C}^{N+1}$ for $G_{N}$. The Landau variety $L^{\prime}=\bigcup_{i=0}^{N} L_{i}^{\prime}$ of $G_{N-1}$ then is identified with $\bigcup_{i=0}^{N-1}\left(L_{i} \cap L_{N}\right) \cup\left(L_{N+1} \cap L_{N}\right) \subset \mathbb{C}^{N+1}$. We choose for $\mathscr{G}_{N-1}$ the base point $B^{\prime}=(1, \varepsilon, \varepsilon, \ldots, \varepsilon, 0) \in \mathbb{C}^{N+1}$. For sufficiently small $\eta$ any element $\gamma$ of $\mathscr{G}_{N-1}$ has a representative $\underline{\gamma}$ which does not come within a distance $\eta$ of $\mathrm{L}^{\prime}$. We can then choose $\varepsilon$ (depending only on $\eta$ ) sufficiently small that for all $t, 0<t \leqq \varepsilon$, the loop $\underline{\gamma}(t)=\left\{(s):\left(s_{0}, \ldots, s_{N-1}\right)\right.$ $\left.\in \mathcal{Y} S_{N}=t\right\}$ does not intersect $L$. We then define $j(\gamma) \in \mathscr{G}_{N}$ to be the element of $\mathscr{G}_{N}$ defined by $\underline{\gamma}(\varepsilon)$. If $\underline{\gamma}^{\prime}$ is another representative of $\gamma$, we may choose the homotopy $H$ between $\underline{\gamma}$ and $\underline{\gamma}^{\prime}$ so that the image of $H$ also does not come within $\eta$ of $L^{\prime} ; H$ then gives rise to a homotopy $H(\varepsilon)$ between $\underline{\gamma}(\varepsilon)$ and $\underline{\gamma}^{\prime}(\varepsilon)$. Thus $j$ is well defined on $\mathscr{G}_{N-1}$.

We remark that

$$
\left.\begin{array}{rlrl}
j\left(\beta_{k}^{\prime}\right) & =\beta_{k} & & (k=1, \ldots N-1) \\
j\left(\beta_{0}^{k \prime}\right) & =\beta_{0}^{k)} & & (k=1, \ldots N-1) \\
j\left[\alpha(\chi)^{\prime}\right] & =\alpha(\chi) \alpha(\chi \cup N) & & \left(\chi \text { a proper subset of } \Omega_{N}\right) .
\end{array}\right\}
$$

\subsection{The Relations Obtained from the Integral Representation}

(i) Homogeneity.

The function $D^{\prime}(s, \alpha)$ is homogeneous and linear in $s$. It follows that the function $I(s, \lambda)$ defined by (2.1.18) is homogeneous in $s$ of degree

$$
-\sum_{i=0}^{N} \lambda_{i}-(N+2)=\mu \text { (say) }
$$

This homogeneity can be expressed as a condition on the representation $\mathscr{L}(\lambda)$. If $w_{\infty}$ denotes the element of $\mathscr{G}_{N}$ defined by a loop circling the point at infinity in a generic line $\ell$ counterclockwise (i.e. circling all the points $L \cap \ell$ clockwise) we have

$$
\mathscr{L}\left(w_{\infty}\right)=c_{\infty} 1
$$

where the constant $c_{\infty}$ is given by

$$
c_{\infty}=\exp [-2 \pi i \mu] .
$$

(ii) Consequences of the Picard-Lefschetz theorem. 
In the group ring $\mathbb{C}\left(\mathscr{G}_{N}\right)$, write

$$
\begin{aligned}
\alpha(\phi) & =1+a, & & \\
\beta_{i} & =1+b_{i} & & 1 \leqq i \leqq N, \\
\beta_{0}^{i)} & =1+b_{0}^{i)} & & 1 \leqq i \leqq N .
\end{aligned}
$$

It can be shown that in a standard form presentation of $I(s, \lambda)$ the points on $L$ used to define the elements $\alpha=\alpha(\phi), \beta_{i}, \beta_{0}^{i)}$ of $\mathscr{G}_{N}$ correspond to a quadratic pinch in the integration space; in the case of $\alpha$ this pinch is simple. We can therefore apply the Picard-Lefschetz theorem. For a simple quadratic pinch and integer exponents for the singularities of the integrand this is given in [8]. The extension to a non-simple quadratic pinch is given in [13], and to non-integral exponents for the singularities of the integrand in [4].

In the present situation we obtain from the theorem the relations

$$
\begin{array}{rlrl}
\mathscr{L}\left(a^{2}\right) & =A \mathscr{L}(a), & \\
\mathscr{L}\left(b_{i}^{2}\right) & =B_{i} \mathscr{L}\left(b_{i}\right) \quad 1 \leqq i \leqq N \\
\mathscr{L}\left[\left(b_{0}^{i}\right)^{2}\right] & =B_{0} \mathscr{L}\left(b_{0}^{i}\right) \quad 1 \leqq i \leqq N,
\end{array}
$$

where the constants $A, B_{i} 0 \leqq i \leqq N$ are given by

$$
\begin{aligned}
1+A & =(-1)^{N+1} \exp [2 \pi i \mu], \\
1+B_{i} & =\exp \left[-2 \pi i \lambda_{i}\right] .
\end{aligned}
$$

Thus

$$
\prod_{i=0}^{N}\left(1+B_{i}\right)=(-1)^{N+1}(1+A)=c_{\infty}^{-1} .
$$

To express the further relations derived from the fact that $\alpha$ corresponds to a simple quadratic pinch we introduce the notion of a Lefschetz element.

Definition 2.3.13. An element $e$ of an associative algebra $A$ is Lefschetz if

$$
e^{2}=E e
$$

and for all $x \in A$

$$
\text { exe is a multiple of } e \text {. }
$$

We then write

$$
\text { exe }=(e \cdot x) e .
$$

Remark. $e \cdot x$ is linear in $x$. If $e, f$ are both Lefschetz

$$
e \cdot f=f \cdot e \text {. }
$$


Thus the dot product defines a symmetric bilinear product on the linear span of the Lefschetz elements.

Now we can state the further relations as:

$$
\mathscr{L}(a) \text { is a Lefschetz element of } \mathscr{A}_{N}(\lambda) \text {. }
$$

The linear functional $\mathscr{L}(a) \cdot x, x \in \mathscr{A}_{N}(\lambda)$, which appears in (2.3.13) is not given by the Picard-Lefschetz theorem but must be determined by exploiting the other relations satisfied by the representation (see $\$ 2.4$ ).

(iii) Localization conditions.

These conditions follow from

Theorem 2.3.15. Suppose $L_{1} \ldots L_{k}$ are Landau varieties intersecting transversely at a point $O$ of the parameter space, so that we may choose local coordinates $u$ with $O$ as origin such that

$$
L_{i}=\left\{(u): u_{i}=0\right\} \quad 1 \leqq i \leqq k .
$$

Choose a base point $B$ in the neighbourhood of $O$, and denote by $\gamma_{1} \ldots \gamma_{k}$ the (mutually commuting) elements of the fundamental group corresponding to loops on $B$ around $L_{1} \ldots L_{k}$, and by

$$
T_{i}=\mathscr{L}\left(\gamma_{i}\right)-1 \quad 1 \leqq i \leqq k
$$

the corresponding discontinuity operations in the monodromy ring. Suppose that $L_{1} \ldots L_{k}$ correspond to quadratic pinches in the integration space on the varieties $P_{1} \ldots P_{k}$, and that for $u=0 P_{1} \ldots P_{k}$ are in general position. Suppose further that

$$
P_{1} \cap \cdots \cap P_{k}=\phi
$$

Then

$$
T_{1} \ldots T_{k}=0 .
$$

Remark. For $k=2$ and $P_{1}, P_{2}$ single points, Theorem 2.3 .15 follows immediately from the localization lemma used in the FFLP proof of the Picard-Lefschetz theorem [8]. We give a sketch of the proof in the general case:

Construct a metric in the integration space with respect to which $P_{1}, \ldots, P_{k}$ are orthogonal, and denote by $N_{i}(\eta)$ the tubular neighbourhood of radius $\eta$ constructed with the aid of this metric. Then for $\eta$ sufficiently small

$$
N_{1}(\eta) \cap \cdots \cap N_{k}(\eta)=\phi .
$$

We can choose $\delta=\delta(\eta)$ sufficiently small, that for $u \in \sigma_{i}$

$$
\sigma_{i}=\left\{(u): u_{j}=\delta \quad j \neq i \quad\left|u_{i}\right| \leqq \delta\right\}
$$


the poles of the integrand are in G.P. outside $N_{i}(\eta)$. Then we may choose

$$
B=(\delta, \delta, \ldots, \delta)
$$

as base point, and take $\gamma_{i}=\partial \sigma_{i} 1 \leqq i \leqq N$. The ambiant isotopy $A_{i}$ for $\gamma_{i}$ may then be chosen to be the identity outside $N_{i}(\eta)$ and inside $N_{i}(\eta)$ to commute with the projection $P_{i}$ of the normal bundle $N_{i}(\eta)$ onto $P_{i}$.

Now let $\underline{h}$ be an arbitrary integration cycle defining a function element $h$. Then if we compute a representative cycle for $T_{1} \ldots T_{k} h$ with the help of the ambiant isotopies $A_{i}$ we obtain successively

$$
\begin{array}{ll} 
& \operatorname{supp} A_{k} \underline{h} \subset N_{k}(\eta) \\
\ldots & \operatorname{supp} A_{j} \ldots A_{k} \underline{h} \subset N_{j}(\eta) \cap \cdots \cap N_{k}(\eta) \\
\text { and so } & \operatorname{supp} A_{1} \ldots A_{k} \underline{h} \subset N_{1}(\eta) \cap \cdots \cap N_{k}(\eta)=\phi, \\
\text { so } & T_{1} \ldots T_{k} h=0 .
\end{array}
$$

$h$ is arbitrary so $T_{1} \ldots T_{k}=0$.

We apply Theorem 2.3.15 to the transverse intersection of $L_{1} \ldots L_{N}$. If $\lambda_{0}, \ldots, \lambda_{N}$ satisfy a certain linear relation we may rewrite (2.1.18) in the momentum space form, $m=2$,

$$
I(s, \lambda)=\int \prod_{i=1}^{N} \frac{d^{2} k_{i}}{\left(s_{l}+k_{i}^{2}\right)^{\lambda_{1}+2}} \delta\left(\sum_{i=1}^{N} k_{i}-p\right) .
$$

Now a point on $L_{i}$ corresponds in the integration space to a quadratic pinch on

$$
P_{i}=\left\{(k): k_{i}=0, \sum_{j=1}^{N} k_{i}-p=0\right\} .
$$

The $P_{i} 1 \leqq i \leqq N$ are in general position, and for $s_{0} \neq 0$, i.e. $p \neq 0$

$$
P_{i} \cap \cdots \cap P_{N}=\phi \text {. }
$$

Hence Theorem 2.3.15 applies, and we obtain the relation

$$
\mathscr{L}\left(b_{1} \ldots b_{N}\right)=0 \text {. }
$$

It is also possible to derive (2.3.19) from the geometry of the pinches corresponding to the $L_{i}$ in the $\alpha$-space, so that (2.3.19) holds for generic $\lambda$. In view of the symmetry of the $\alpha$-space representation (2.1.18) noted in $\S 2.1$ we have also for the transverse intersection of $L_{i} i \neq j, L_{0}^{j)}$

$$
\mathscr{L}\left(b_{1} \ldots \hat{b}_{J} \ldots b_{N} b_{0}^{j)}\right)=0 \text {. }
$$

Finally we apply Theorem 2.3 .15 to the real self-intersections of $L_{N+1}$ (cf. (2.2.9)) and obtain

$$
\left.\begin{array}{l}
\mathscr{L}\left[a\left(\chi_{1}\right) a\left(\chi_{2}\right)\right]=0 \\
\text { for all proper subsets } \chi_{1}, \chi_{2} \text { of } \Omega \text { such that } \\
\qquad \chi_{1} \varangle \chi_{2}, \chi_{2} \varangle \chi_{1}, \chi_{1} \cup \chi_{2} \neq \Omega
\end{array}\right\}
$$


where

$$
a(\chi)=\alpha(\chi)-1
$$

(iv) Physical sheet conditions.

Denote by $\Psi$ the vector in $V_{N}$ defined by the contour of integration $\Delta^{\prime \prime}$ in (2.1.18). $\Psi$ is called the physical sheet. By examining whether the corresponding point $P$ on $L$ corresponds to a pinch of $\Delta^{\prime \prime}$ we may decide whether $\Psi$ is invariant under a given element of $\mathscr{G}_{N}$ (for generic $\lambda$ there are no polar singularities so $\Psi$ is invariant if it is nonsingular at $P$ ). This gives the physical sheet conditions

$$
\begin{array}{rl}
\mathscr{L}(a) \Psi \neq 0, & \\
\mathscr{L}[a(\chi)] \Psi=0 & \chi \neq \phi, \\
\mathscr{L}\left(b_{0}^{i)}\right) \Psi=0 & 1 \leqq i \leqq N .
\end{array}
$$

Finally an element of $\mathscr{A}_{N}$ must be determined by its action on the vectors of $V_{N}=\mathscr{A}_{N} \Psi$, i.e.

$$
y \in \mathscr{A}_{N}, y \mathscr{A}_{N} \Psi=0 \Rightarrow y=0 .
$$

It turns out that the ring defined by all the relations except the physical sheet conditions is already isomorphic with a complete matrix ring. We do not therefore obtain any additional relations from (2.3.25). The role of the physical sheet conditions is just to give a matrix representation of the abstract ring by singling out the vector $\Psi$, and to guarantee that the tacnode points of Fig. 10 are effective intersections (see $\S 2.4$ ), that is,

$$
\mathscr{L}\left[a b_{i}\right] \neq \mathscr{L}\left[b_{i} a\right] \quad(i=1 \ldots N)
$$

Eqs. (2.3.2), (2.3.7)-(2.3.9), (2.3.14), (2.3.19)-(2.3.25) are the relations which we use in $\$ 2.4$. Note that the relations listed in this section do not include the Cutkosky-Steinmann relations used in [11]. These relations depend on the vanishing of certain intersection numbers of cycles in the integration space, algebraically not geometrically (the cycles are not disjoint), and do not persist in the generic case.

\subsection{Derivation of the Monodromy Ring $\mathscr{A}_{N}$ in the Generic Case}

Let $\mathscr{M}$ denote the set of all proper subsets of $\Omega$. For any $\chi \in \mathscr{M}$, define

(with $b(\phi)=1$ ).

$$
b(\chi)=\prod_{i \in \chi} b_{i}
$$


Lemma 2.4.1. For any $\chi \in \mathscr{M}$

$$
\mathscr{L}(a b(\chi) a)=M(\chi) \mathscr{L}(a)
$$

where

$$
M(\chi)=(-)^{|\chi|}(1+A)-\prod_{i \in \chi}\left(1+B_{i}\right)
$$

(i.e., in the notation of Definition 2.3.13,

$$
\mathscr{L}(a) \cdot \mathscr{L}[b(\chi)]=M(\chi)) .
$$

Proof. For $|\chi|=0$, this is an immediate consequence of (2.3.7). For $\chi=\{i\}$, we apply $\mathscr{L}$ to the bicommutation relation

yielding

$$
\left(\alpha \beta_{i}\right)^{2}=\left(\beta_{i} \alpha\right)^{2}
$$

$$
\left[\mathscr{L}(a) \cdot \mathscr{L}\left(b_{i}\right)-M(\{i\})\right] \mathscr{L}\left[a b_{i}-b_{i} a\right]=0 .
$$

(2.4.2) then follows from (2.3.26).

Suppose now that we have verified (2.4.2) for all $|\chi| \leqq m<N-2$, and suppose $|\eta|=m+1$, with $\eta=i \cup \chi$. Eq. (2.3.21) implies

$$
\mathscr{L}\left[a \beta_{i}^{-1} \prod_{j \in \chi} \beta_{j} a\right]=0 ;
$$

applying the induction assumption to this formula gives (2.4.2).

Lemma 2.4.4 [Reduction of the word at infinity]: $\mathscr{L}\left(\beta_{0}^{i)^{-1}}\right)$

$$
=c_{\infty} \mathscr{L}\left\{\beta_{1} \ldots \beta_{N}+\beta_{i}\left[\sum_{\substack{\psi, x<\Omega_{1} \\ \psi \cap \chi<\phi}} \prod_{\chi} \beta_{j} a \prod_{\psi} \beta_{j} \prod_{\Omega_{i}-(\chi \cup \psi)}\left[-\left(2+B_{j}\right)\right]\right]\right\} .
$$

Proof. From (2.2.10) we have

$$
\beta_{0}^{i)^{-1}}=w_{\infty}^{(i)} \beta_{i} \alpha(\phi) \prod_{j \neq i} \alpha(\{j\}) \ldots \alpha\left(\Omega_{i}\right) \prod_{j \neq l} \beta_{j} .
$$

We apply $\mathscr{L}$ to this and use (2.3.2). Write $\alpha(\chi)=1+a(\chi)$ and expand

All products

$$
\mathscr{L}\left[\alpha(\phi) \prod_{j \neq i} \alpha(\{j\}) \ldots \alpha\left(\Omega_{i}\right)\right] .
$$

$$
\mathscr{L}\left[\alpha\left(\chi_{1}\right) \alpha\left(\chi_{2}\right) \ldots \alpha\left(\chi_{k}\right)\right]
$$

vanish by (2.3.21) unless $\chi_{1} \subset \chi_{2} \subset \cdots \subset \chi_{k}$. Then using

$$
a(\chi)=\prod_{\chi} \beta_{j} a \prod_{\chi} \beta_{j}^{-1}
$$


(2.4.6) becomes

$$
\begin{aligned}
& \mathscr{L}\left[\beta_{0}^{\left.i)^{-1}\right]}\right. \\
& =c_{\infty}\left\{\beta_{1} \ldots \beta_{N}+\beta_{i} \sum_{k=1}^{N} \sum_{\chi_{1}, \chi_{2} \ldots \chi_{k}} \prod_{\chi_{1}} \beta_{j} a \prod_{\chi_{2}} \beta_{j} \ldots \prod_{\chi_{k}} \beta_{j} a \prod_{\left(\Omega_{2}-\chi_{1} \cup \cdots \cup \chi_{k}\right)} \beta_{j}\right\},
\end{aligned}
$$

where $\chi_{1} \ldots \chi_{k}$ are disjoint subsets of $\Omega_{i}$ with $\chi_{j} \neq \phi$ for $j>1$. Set $\chi=\chi_{1}$, $\psi=\Omega_{i}-\left(\chi_{1} \cup \cdots \cup \chi_{k}\right)$. From Lemma (2.4.2) we have

so that

$$
\mathscr{L}\left[a \prod_{\chi} \beta_{j} a\right]=-\prod_{\chi}\left(2+B_{j}\right) \mathscr{L}(a), \quad(\chi \in \mathscr{M})
$$

$$
\mathscr{L}\left[a \prod_{\chi_{2}} \beta_{j} a \ldots a \prod_{\chi_{k}} \beta_{j} a\right]=(-)^{k} \prod_{\Omega_{t}-(x \cup \psi)}\left(2+B_{j}\right) \mathscr{L}(a) .
$$

But if $\eta$ is any set of $m>0$ elements, one has

$$
\sum_{P}(-)^{|P|}=(-)^{m}
$$

where the sum runs over all ordered partitions $P$ of $\eta$ into $|P|$ sets. Thus we may do the sum over $\chi_{2} \ldots \chi_{k}$ in (2.4.7) for fixed $\chi, \psi$; this yields (2.4.5).

We define $I_{N} \subset \mathbb{C}\left(\mathscr{G}_{N}\right)$ to be the two-sided ideal generated by the elements

$$
\begin{aligned}
& a(\chi) a(\psi) \quad(\chi \not \psi, \psi \nsubseteq \chi, \chi \cup \psi \neq \Omega) \\
& b_{1} \ldots b_{N} \quad \chi, \psi \in \mathscr{M} \\
& \left.\begin{array}{l}
w_{\infty}^{i)}-c_{\infty} 1 \\
b_{i}^{2}-B_{i} b_{i}
\end{array}\right\} \quad 1 \leqq i \leqq N \\
& b_{0}^{i{ }^{2}}-B_{0} b_{0}^{i)} \\
& a^{2}-A a \\
& a b(\chi) a-M(\chi) a \quad \chi \in \mathscr{M}
\end{aligned}
$$

and form the quotient ring

$$
R_{N}=\mathbb{C}\left(\mathscr{G}_{N}\right) / I_{N} .
$$

We denote by $q_{N}$ the natural projection $\mathbb{C}\left(\mathscr{G}_{N}\right) \rightarrow R_{N}$ and indicate the image under $q_{N}$ of an element of $\mathbb{C}\left(\mathscr{G}_{N}\right)$ by underlining. We have shown that the elements of $I_{N}$ lie in the kernel of $\mathscr{L}: \mathbb{C}\left(\mathscr{G}_{N}\right) \rightarrow L\left(V_{N}\right)$, so there is a natural homomorphism $\mathscr{L}: R_{N} \rightarrow L\left(V_{N}\right)$.

Lemma 2.4.10. $R_{N}$ is finite dimensional.

Proof. $\mathbb{C}\left(\mathscr{G}_{N}\right)$ is spanned by all products of the elements $1, \underline{b}_{i}, \underline{b}_{0}^{i)}, \underline{a}$. The reduction of the word at infinity (Lemma 2.4.4), which is also valid in $R_{N}$ (since only the vanishing of the elements (2.4.8) was used in its derivation), enables us to eliminate the $\underline{b}_{0}^{i}$. We then obtain the following 
finite set of elements spanning $R_{N}$

$$
\underline{b}(\chi), \underline{b}\left(\chi_{1}\right) \underline{a} \underline{b}\left(\chi_{2}\right), \quad \chi, \chi_{1}, \chi_{2} \in \mathscr{M} .
$$

Lemma 2.4.12. $\underline{b}\left(\Omega_{i}\right)$ is Lefschetz.

Proof. It is sufficient to prove that

$$
\underline{b}\left(\Omega_{i}\right) \underline{x} \underline{b}\left(\Omega_{i}\right)-\left(\underline{b}\left(\Omega_{i}\right) \cdot \underline{x}\right) \underline{b}\left(\Omega_{i}\right)=0
$$

for each $\underline{x}$ in the set (2.4.11) and some $\underline{b}\left(\Omega_{i}\right) \cdot \underline{x} \in \mathbb{C}$. This follows easily for all $\underline{x}$ in (2.4.11) once it is established for $\underline{x}=\underline{a}$. In this case the symmetry of the product gives $\underline{b}\left(\Omega_{i}\right) \cdot \underline{a}=M\left(\Omega_{i}\right)$ so we must prove

Now

$$
\underline{y}=\underline{b}\left(\Omega_{i}\right) \underline{a} \underline{b}\left(\Omega_{i}\right)-M\left(\Omega_{i}\right) \underline{b}\left(\Omega_{i}\right)=0 .
$$

From (2.4.4) we obtain

$$
\begin{aligned}
\underline{b}_{j} \underline{y} & =B_{j} \underline{y} \quad j=1 \ldots \hat{i} \ldots N \\
\underline{b}_{i} \underline{y} & =0 \\
\underline{a} \underline{y} & =0 .
\end{aligned}
$$

$$
\underline{B}_{0}^{i)^{-1}} \underline{y}=c_{\infty} \prod_{j \neq i}\left(1+B_{j}\right) \underline{y} .
$$

But $\underline{\beta}_{0}^{i)^{-1}} \underline{y}=f y$ implies $y=0$ or $f=\left(1+B_{0}\right)^{-1}$ or 1 . For generic $\lambda$ $f=c_{\infty} \prod_{j \neq i}\left(1+B_{j}\right) \neq\left(1+B_{0}\right)^{-1}$ or 1 so $y=0$.

Lemma 2.4 .13 (reduction of the identity). The element $\underline{1}=\underline{b}(\phi) \in R_{N}$ is linearly dependent on the elements $\underline{b}(\chi)(\chi \neq \phi)$ and $\underline{b}(\chi) \underline{a} \underline{b}(\psi)$. Specifically, if

then

$$
\begin{aligned}
Y(\chi) & =\prod_{\chi}\left(-\frac{1}{B_{i}}\right) \quad(Y(\phi)=1), \\
D & =-\sum_{\chi \in \mathscr{M}} M(\chi) Y(\chi),
\end{aligned}
$$

$$
\underline{h}=\sum_{\chi \in \mathscr{M}} Y(\chi) \underline{b}(\chi)+\sum_{\chi, \psi} \frac{Y(\chi) Y(\psi)}{D} \underline{b}(\chi) \underline{a} \underline{b}(\psi)=0 .
$$

Proof.Direct calculation gives

$$
\underline{a} \underline{h}=\underline{b}_{i} \underline{h}=0 \quad 1 \leqq i \leqq N .
$$

Then from (2.4.4) $\underline{\beta}_{0}^{i)^{-1}} \underline{h}=c_{\infty} \underline{h}$. Since $c_{\infty} \neq\left(1+B_{0}\right)^{-1}$ or 1 this implies $\underline{h}=0$ (cf. proof of 2.4 .12 ).

We now give the main result of this section.

Theorem 2.4.17 [Determination of $\mathscr{A}_{N}$ ]. The dimension of $V_{N}$ is $2^{N}-1$, and $\mathscr{L}: R_{N} \rightarrow L\left(V_{N}\right)$ is an isomorphism onto. Thus $R_{N} \cong \mathscr{A}_{N}$, which is a complete matrix algebra of dimension $2^{N}-1$. 
Proof. Recall that $\Psi$ denotes the physical sheet. From Lemma 2.4.10, $V_{N}$ is spanned by the elements

$$
\mathscr{L}[\underline{b}(\chi)] \Psi, \underline{L}\left[\underline{b}\left(\chi_{1}\right) \underline{a} \underline{b}\left(\chi_{2}\right)\right] \Psi \quad\left(\chi, \chi_{1}, \chi_{2} \in \mathscr{M}\right) .
$$

The following Lemma shows that $V_{N}$ is actually spanned by the $2^{N}-1$ elements $\underline{L}[\underline{b}(\chi)] \Psi, \chi \in \mathscr{M}$.

\section{Lemma 2.4 .18}

$$
\begin{gathered}
\mathscr{L}[\underline{a}(\chi)] \Psi=\prod_{\chi}\left(1+B_{i}\right) \underline{L}(\underline{a}) \Psi, \\
\mathscr{L}[\underline{b}(\chi)(1+\underline{a})] \Psi=\sum_{\psi \supset} \sum_{\chi, \psi \in \mathcal{M}} \frac{(-)^{|\psi-\chi|} c_{\infty}^{-1}}{\prod_{\psi}\left(1+B_{i}\right)} \mathscr{L}[\underline{b}(\chi)] \Psi .
\end{gathered}
$$

Proof. Eq. (2.3.23) implies

$$
\mathscr{L}\left[\underline{a} \prod_{\eta} \underline{B}_{i}^{-1}\right] \Psi=0 \quad(\eta \in \mathscr{M}, \eta \neq \phi) .
$$

Now (2.4.19) is trivially true for $\chi=\phi$; it then follows from (2.4.21) by induction on $|\chi|$, using $\underline{\beta}_{i}^{-1}=1-\frac{\underline{b}_{i}}{1+B_{i}}$.

Since $\beta_{j}(j \neq i)$ commutes with $\beta_{0}^{i}$, we may rewrite (2.4.6) as

$$
\beta_{0}^{i)^{-1}}=\prod_{j \neq i} \beta_{j} w_{\infty}^{i} \beta_{i} \alpha(\phi) \ldots \alpha\left(\Omega_{i}\right) .
$$

Using (2.3.23) and (2.3.24), we have

$$
\mathscr{L}\left(\prod_{i=1}^{N} \underline{\beta}_{i} \underline{\alpha}\right) \Psi=c_{\infty}^{-1} \Psi
$$

and multiplying (2.4.22) by $\mathscr{L}[\underline{b}(\chi)]$ gives

$$
\prod_{\eta}\left(1+B_{j}\right) \mathscr{L}\left\{\left[\sum_{\psi \supset} \underline{\eta}, \underline{b} \in \underline{M}(\psi)\right](1+\underline{a})\right\} \Psi=c_{\infty}^{-1} \Psi .
$$

Taking $\eta=\Omega_{i}$ in (2.4.23) verifies (2.4.20) for $\chi=\Omega_{i}$. (2.4.20) follows for any $\chi_{0}$ from (2.4.23) (taking $\eta=\chi_{0}$ ) if we assume, inductively, that (2.4.20) holds for any $\chi \supset \chi_{0}$.

We now return to the proof of the main theorem. We write $\Psi(\varrho)$ $=\mathscr{L}(b(\varrho)) \Psi$. From Lemma 2.4.18 we obtain the relation $\mathscr{L}(b(\psi) a b(\chi)) \Psi(\varrho)$

$$
\begin{aligned}
& =\left\{\begin{array}{l}
0 \text { if } \varrho \cup \chi=\Omega \\
\prod_{\varrho \cap \chi} B_{i} \prod_{\varrho \cup \chi}\left(1+B_{i}\right)\left[-b(\psi)+\sum_{\eta \supset \psi} \frac{(-1)^{|\eta-\psi|} c_{\infty}^{-1} b(\eta)}{\prod_{\eta}\left(1+B_{i}\right)}\right] \Psi
\end{array}\right. \\
& =v_{\varrho}^{\chi} \sum_{\eta \in \mathcal{M}} u_{\eta}^{\psi} \Psi(\eta) \text {, }
\end{aligned}
$$


where the coefficients $v_{\varrho}^{\chi}, u_{\eta}^{\psi}$ are given by

$$
\begin{aligned}
& v_{\varrho}^{\chi}= \begin{cases}0 & \varrho \cup \chi=\Omega \\
\prod_{\varrho \cap \chi} B_{i} \prod_{\varrho \cup \chi}\left(1+B_{i}\right) & \text { otherwise }\end{cases} \\
& u_{\eta}^{\psi}= \begin{cases}0 \text { unless } \eta \supset \psi \\
-1+\frac{c_{\infty}^{-1}}{\prod_{\psi}\left(1+B_{i}\right)} \quad \eta=\psi \\
(-1)^{|\eta-\psi|} \frac{c_{\infty}^{-1}}{\prod_{\eta}\left(1+B_{i}\right)} & \eta \supsetneqq \psi .\end{cases}
\end{aligned}
$$

We may regard $(2.4 .25),(2.4 .26)$ as defining two sets of $2^{N}-1$ vectors $v(\chi) \chi \in \mathscr{M}, u(\psi) \psi \in \mathscr{M}$ in the vector space

$$
\mathbb{C}^{2^{N}-1}=\left\{(x)=\left(x_{\varrho}\right) \varrho \in \mathscr{M}\right\} .
$$

We claim that for generic $\lambda$ each of these sets is a basis for $\mathbb{C}^{2^{N}-1}$.

For the set $\{u(\psi)\}$ this follows immediately from (2.4.26) since the coefficient matrix $[u]=\left[u_{\eta}^{\psi}\right]$ is "triangular", i.e. $u_{\eta}^{\psi}=0$ for $\eta \nsubseteq \psi, u_{\eta}^{\eta} \neq 0$. For the set $\{v(\chi)\}$ we argue as follows. It is sufficient to show that the vectors $w(\chi)$ defined by

$$
w_{\varrho}^{\chi}=\left\{\begin{array}{l}
0 \quad \varrho \cup \chi=\Omega \\
\prod_{\varrho \cap \chi} B_{i} \prod_{\varrho-\chi}\left(1+B_{i}\right) \quad \text { otherwise }
\end{array}\right.
$$

are linearly independent since

$$
w(\chi)=\prod_{\chi}\left(1+B_{i}\right)^{-1} v(\chi),
$$

i.e., to show that the corresponding matrix $[w]=\left[w_{\varrho}^{\chi}\right]$ has non-zero determinant. This determinant has degree

$$
\leqq \sum_{\varrho \in \mathscr{M}}|\varrho|=\sum_{k=0}^{N-1} k^{N}\left(\begin{array}{l}
N \\
k
\end{array}\right)=N\left(2^{N-1}-1\right)
$$

in the $B_{i}$. It will suffice to show that there is a non-zero term of this degree. To do this we may specialize to the case in which all the $B_{i}$ are equal. Then the leading term in $B$ of $\operatorname{det}[w]$ is

where

$$
B^{N\left(2^{N-1}-1\right)} \operatorname{det}[y]
$$

$$
y_{\varrho}^{\chi}= \begin{cases}0 & \varrho \cup \chi=\Omega \\ 1 & \text { otherwise }\end{cases}
$$


From the vectors $y(\chi)$ we can form vectors

which have components

$$
z(\chi)=\sum_{\eta \subset \chi}(-1)^{|\eta|} y(\eta)
$$

$$
z_{\eta}^{\chi}=\delta_{\chi \phi}-(-1)^{|(\Omega-\varrho)|} \delta_{\chi, \Omega-\varrho}
$$

and are manifestly linearly independent.

Since the sets $\{u(\psi)\},\{v(\psi)\}$ form bases for $\mathbb{C}^{2^{N}-1}$ for generic $\lambda$ there exists coefficients $C_{\eta}^{\chi}(\lambda), D_{\eta}^{\chi}(\lambda)$ such that

Then

$$
\begin{aligned}
& \sum_{x \in \mathcal{M}} C_{\eta}^{\chi} v_{\varrho}^{\alpha}=\delta_{\eta \varrho}, \\
& \sum_{\chi \in \mathcal{M}} D_{\eta}^{\alpha} u_{\varrho}^{\alpha}=\delta_{\eta \varrho} .
\end{aligned}
$$

satisfies

$$
f_{\eta \varrho}=\sum_{\chi, \psi \in \mathscr{M}} \mathscr{L}[b(\psi) a b(\chi)] C_{\eta}^{\chi} D_{\varrho}^{\psi} \in \mathscr{A}_{N}(\lambda)
$$

$$
f_{\eta \varrho} \Psi_{\kappa}=\delta_{\eta \kappa} \Psi_{\varrho} .
$$

If for some coefficients $g_{\varrho} \in \mathbb{C}$ we have

$$
\sum g_{\varrho} \Psi_{\varrho}=0
$$

we may apply $f_{\eta \phi}$ to this relation and use (2.4.27) to obtain

$$
g_{\eta} \Psi=0 \text {. }
$$

But $\Psi \neq 0$ so $g_{\eta}=0$ for all $\eta$, i.e. the $\Psi_{e}$ are linearly independent. Moreover the $f_{\eta \varrho}$ are $\left(2^{N}-1\right)^{2}$ linearly independent elements of $\mathscr{A}_{N}(\lambda)$.

This completes the proof of the first part of the main theorem.

There remains to show only that $\underline{\mathscr{L}}: R_{N} \rightarrow \mathscr{A}_{N}$ has zero kernel; we do this by showing that the dimension of $R_{N}$ is at most $\left(2^{N}-1\right)^{2}$. In the course of the argument we will also prove the important isomorphism theorem mentioned in the Introduction. We proceed by induction on $N$ : for $N=2$, Lemmas (2.4.10), (2.4.12), and (2.4.13) imply that $R_{N}$ is spanned by the nine elements

$$
\underline{b}\left(\chi_{1}\right) \underline{a} \underline{b}\left(\chi_{2}\right) \quad\left[\chi_{1}, \chi_{2}=\phi,\{1\}, \text { or }\{2\}\right],
$$

thus $R_{2} \cong \mathscr{A}_{2}$. We assume inductively that $R_{N-1}$ is spanned by the elements

$$
\underline{b}\left(\chi_{1}\right) \underline{a} \underline{b}\left(\chi_{2}\right) \quad\left(\chi_{1}, \chi_{2} \in \mathscr{M}\right) .
$$

Define $P \in R_{N}$ by $P=\underline{b}_{N} / B_{N}$ (so that $P^{2}=P$ ), and let $S_{N} \subset R_{N}$ denote the sub-algebra $S_{N}=P R_{N} P$. There is a natural map $p: R_{N} \rightarrow S_{N}$ given by 
$p(\underline{x})=P \underline{x} P ; p$ is a linear map but is not a ring homomorphism. However, if

$$
Y_{N}=\left\{\underline{x} \in R_{N} \mid \underline{x} \text { commutes with } P\right\}
$$

then $p \mid Y_{N}$ is a ring homomorphism.

Now recall $(\$ 2.2)$ the homomorphism $j: \mathscr{G}_{N-1} \rightarrow \mathscr{G}_{N}$; this extends directly to a ring homomorphism of $\mathbb{C}\left(\mathscr{G}_{N-1}\right)$ into $\mathbb{C}\left(\mathscr{G}_{N}\right)$ which we also denote by $j$. From (2.2.11) and (2.2.5)-(2.2.8) we see that the image $j\left(\mathscr{G}_{N-1}\right) \subset \mathscr{G}_{N}$ commutes with $\beta_{N}$; therefore the image $q_{N} j\left[\mathbb{C}\left(\mathscr{G}_{N}\right)\right] \subset R_{N}$ is contained in $Y_{N}$ and we may define a ring homomorphism

$$
k_{N}: \mathbb{C}\left(\mathscr{G}_{N-1}\right) \rightarrow S_{N}
$$

by $k_{N}=p q_{N} j$.

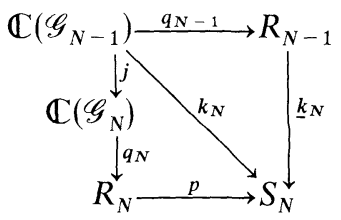

Lemma 2.4.28. The map $k_{N}$ is onto $S_{N}$. The ideal $I_{N-1}$ of $\mathbb{C}\left(\mathscr{G}_{N-1}\right)$ is contained in the kernel of $k_{N}$, so that $k_{N}$ may be factored through a map

$$
\underline{k}_{N}: R_{N-1} \rightarrow S_{N}
$$

with $k_{N}=\underline{k}_{N} q_{N-1} \cdot \underline{k}_{N}$ is an isomorphism.

Proof. We denote by primes variables referring to the graph $G_{N-1}$. Note that

but that

$$
B_{i}^{\prime}=B_{i} \quad(i=0 \ldots N-1)
$$

$$
A^{\prime}=-1-\frac{1+\mathrm{A}}{1+B_{N}} \text {. }
$$

The verifications of the first two statements of the Lemma are straightforward. $\underline{k}_{N}$ is an isomorphism because, by induction, $R_{N-1}$ is a simple ring, and $S_{N}$ is not trivial.

Now our induction assumption and Lemma (2.4.28) imply that any element $\underline{b}(\chi) \in R_{N}$, with $N \in \chi$, may be expressed as a linear combination of the elements

$$
\underline{b}\left(\chi_{1}\right) \underline{a} \underline{b}\left(\chi_{2}\right) \quad\left(\chi_{1}, \chi_{2} \in \mathscr{M}\right) .
$$

Clearly, this also holds for any $\underline{b}(\chi)$ with $\chi$ non-empty. An application of Lemma (2.4.13) then completes the proof.

It is interesting to note that if we require that the representation $\mathscr{L}$ of $\mathscr{G}_{N}$ satisfy all conditions of $\S 2.3$, but consider the constants $c_{\infty}, A$, and $B_{i}$ of (2.3.2) and (2.3.7)-(2.3.9) as independent variables (not connected with the $\lambda$ 's), the relations imply that these constants must satisfy (2.3.12). 


\section{$\S 3$. Specialization of Parameters}

\subsection{Introduction}

In this section we consider three specializations of the generic integral (2.1.18) studied in $\S 2$. The first of these is the convergent integral (2.1.1) with the dimension of space-time $m$ set equal to two. (The convergent case $m=3, N=2$ could be studied similarly.) The second is the integral obtained from (2.1.18) by setting all the masses equal; we also study (2.1.1) in this equal mass case. Finally, we study the integrals obtained from (2.1.18) and (2.1.1) by setting some of the masses equal to zero.

In $\$ 3.2$ we establish certain algebraic results which we will need in this analysis. These are applied in $\S 3.3-\S 3.5$.

\subsection{Rank of the Matrix $v$}

In (2.4.25) we defined a $\left(2^{N}-1\right) \times\left(2^{N}-1\right)$ matrix $v=\left[v_{\varrho}^{\chi}\right](\chi, \varrho \in \mathscr{M})$. In this section we determine the row rank of this matrix when the $\lambda_{i}$ 's have values corresponding to (2.1.1) and the set of linear relations which hold between the row vectors $v^{\chi}$. In this case $B_{i}=0(i=1, \ldots N)$, and (2.2.25) reduces to

$$
v_{\varrho}^{\chi}= \begin{cases}0 & \text { if } \chi \cup \varrho=\Omega \text { or } \quad \chi \cap \varrho \neq \phi, \\ 1 & \text { otherwise }\end{cases}
$$

Note that $v_{\varrho}^{\chi}=0$ if $|\chi|+|\varrho| \geqq N$.

Lemma 3.2.2. For any $\chi, \varrho \in \mathscr{M}$ with $|\varrho|<N-|\chi|$, and any $k$ with $|\varrho| \leqq k<N-|\chi|$,

$$
v_{Q}^{\chi}=\left(\begin{array}{r}
N-|\chi|-|\varrho| \\
k-|\varrho|
\end{array}\right) \sum_{\substack{\psi>\varrho \\
|\psi|=k}} v_{\psi}^{\chi} .
$$

Proof. Clear.

The matrix $v$ may be partitioned into submatrices

$$
v_{j}^{k}=\left\{v_{\varrho}^{\chi}|| \chi|=k,| \varrho \mid=j\right\} .
$$

We have already noted that $v_{j}^{k}=0$ if $k+j \geqq N$. We will be interested in particular in the submatrices $v_{j}^{k}$ with $k+j=N-1$.

Lemma 3.2.3. The row rank of $v$ is equal to the sum of the row ranks of the matrices $v_{j}^{k}$ with $k+j=N-1$.

Proof. Suppose that

$$
\sum_{|\chi|=k} a_{\chi} v_{\varrho}^{\chi}=0 \quad(|\varrho|=N-k-1)
$$


is a linear relation on the rows of $v_{N-k-1}^{k}$. Then by Lemma 3.2.2, this relation holds for all $\varrho$; that is, it gives a relation on the rows of $v$. Conversely, let

$$
\sum_{\chi \in \mathscr{M}} a_{\chi} v_{\varrho}^{\chi}=0 \quad(\varrho \in \mathscr{M})
$$

be a relation on the rows of $v$. Then for $|\varrho|=N-1$, this reduces to

$$
\sum_{|\chi|=0} a_{\chi} v_{\varrho}^{\chi}=0 \text {. }
$$

Again from Lemma 3.2.2, this holds for all $\varrho$, so that (3.2.5) becomes

By induction we obtain

$$
\sum_{|x|>0} a_{x} v_{Q}^{\chi}=0 \text {. }
$$

$$
\sum_{|\chi|=k} a_{\chi} v_{Q}^{\chi}=0
$$

for all $k \leqq N-1$; that is, the relation (3.2.5) is the sum of relations on the rows of the matrices $v_{N-k-1}^{k}$.

Lemma 3.2.6. The matrix $v_{j}^{k}(k+j \leqq N-1)$ has maximum possible rank, i.e.

$$
\operatorname{rank} v_{j}^{k}=\min \left[\left(\begin{array}{c}
N \\
k
\end{array}\right),\left(\begin{array}{c}
N \\
j
\end{array}\right)\right] .
$$

Proof. Since $v$ is symmetric, we may assume without loss of generality that $\left(\begin{array}{l}N \\ j\end{array}\right) \leqq\left(\begin{array}{l}N \\ k\end{array}\right)$. We proceed by induction on $N$; the result is clear for $N=2$. For the induction step we have two cases:

Case 1. $\left(\begin{array}{c}N \\ j\end{array}\right)<\left(\begin{array}{l}N \\ k\end{array}\right)$.

We partition $v_{j}^{k}(N)$ into 4 submatrices $w_{1} \ldots w_{4}$

$$
\begin{array}{ll}
w_{1}=\left[v_{\varrho}^{\chi}\right] 1 \in \chi, 1 \notin \varrho, & w_{2}=\left[v_{\varrho}^{\chi}\right] 1 \in \chi, 1 \in \varrho, \\
w_{3}=\left[v_{\varrho}^{\chi}\right] 1 \notin \chi, 1 \notin \varrho, & w_{4}=\left[v_{\varrho}^{\chi}\right] 1 \notin \chi, 1 \in \varrho .
\end{array}
$$

Note that $w_{2}=0$, and $w_{1}=v_{j}^{k-1}(N-1), w_{4}=v_{j-1}^{k}(N-1)$.

By the induction assumption both $w_{1}$ and $w_{4}$ have maximum possible rank. The assumption of case $1\left(\begin{array}{c}N \\ j\end{array}\right)<\left(\begin{array}{l}N \\ k\end{array}\right)$ implies

so

$$
\left(\begin{array}{c}
N-1 \\
j
\end{array}\right) \leqq\left(\begin{array}{c}
N-1 \\
k-1
\end{array}\right) \text { and }\left(\begin{array}{c}
N-1 \\
j-1
\end{array}\right) \leqq\left(\begin{array}{c}
N-1 \\
k
\end{array}\right)
$$

$$
\operatorname{rank} w_{1}=\left(\begin{array}{c}
N-1 \\
j
\end{array}\right) \quad \operatorname{rank} w_{4}=\left(\begin{array}{c}
N-1 \\
j-1
\end{array}\right) .
$$


This gives $\operatorname{rank} v_{j}^{k}(N)=\left(\begin{array}{c}N-1 \\ j-1\end{array}\right)+\left(\begin{array}{c}N-1 \\ j\end{array}\right)=\left(\begin{array}{c}N \\ j\end{array}\right)$, which completes the induction step.

Case 2. $\left(\begin{array}{c}N \\ j\end{array}\right)=\left(\begin{array}{l}N \\ k\end{array}\right)\left[\right.$ i.e., $\left.j=k \leqq\left[\begin{array}{c}N \\ 2\end{array}\right]\right]$.

We partition $v_{j}^{k}(N)$ into 4 submatrices $w_{1} \ldots w_{4}$ as in case 1 , and note that $w_{3}$ is nonsingular, since $w_{3}=v_{j}^{k}(N-1)$ unless $N$ odd and $k=\left[\frac{N}{2}\right]$, when

$$
v_{\varrho}^{\chi}=\delta_{\varrho}^{\Omega_{1}-\chi} \quad \text { for } \quad 1 \notin \chi, 1 \notin \varrho \quad|\chi|=|\varrho|=\left[\frac{N}{2}\right] .
$$

We now define a new matrix $x$ by $x_{\varrho}^{\chi}=v_{0}^{\chi}$ for $1 \notin \chi$ and

$$
x_{Q}^{\chi}=(N-2 k) v_{Q}^{\chi}-\sum_{j \notin \chi} v_{Q}^{\chi-\{1\} \cup\{\}\}} \text { for } 1 \in \chi .
$$

Then if $1 \in \chi, x_{\varrho}^{\chi}=0$ for $1 \notin \varrho$, and for $1 \in \varrho$

$$
x_{\varrho}^{\chi}=-(N-2 k+1) v_{\varrho}^{\chi-\{1\}} .
$$

We thus obtain a partitioned matrix $x$ equivalent to $v_{j}^{k}$ whose blocks $x_{1} \ldots x_{4}$ satisfy

$$
\begin{aligned}
& x_{1}=0, \quad x_{2}=-(N-2 k+1) v_{j-1}^{k-1}(N-1), \\
& x_{3} \text { is nonsingular. }
\end{aligned}
$$

By the induction assumption the square matrix $x_{2}$ has maximum possible rank. This gives $\operatorname{rank} v_{j}^{k}(N)=\left(\begin{array}{c}N-1 \\ k\end{array}\right)+\left(\begin{array}{c}N-1 \\ k-1\end{array}\right)=\left(\begin{array}{c}N \\ k\end{array}\right)$.

Lemma 3.2.6 implies

$$
\operatorname{rank} v=2^{N}-\left(\begin{array}{c}
N \\
{[N / 2]}
\end{array}\right) \text {. }
$$

Note that for $\left(\begin{array}{l}N \\ k\end{array}\right) \leqq\left(\begin{array}{c}N \\ N-1-k\end{array}\right)$ the rows of the matrix $v_{N-1-k}^{k}$ are linearly independent. For $\left(\begin{array}{l}N \\ k\end{array}\right)>\left(\begin{array}{c}N \\ N-1-k\end{array}\right)$ they satisfy $\mid\left(\begin{array}{c}N \\ N-1-k\end{array}\right)$ $-\left(\begin{array}{l}N \\ k\end{array}\right)$ independent linear relations, and by Lemma 3.2.3 these relations also hold as relations on the complete rows $v^{\chi}$ with $|\chi|=k$.

We now write down a set of linear relations on the rows of $v$ and show that any linear relation on the rows of $v$ is a consequence of these.

Lemma 3.2.8. For any integer $p \leqq\left[\frac{N}{2}\right]$ and any subset of $2 p$ distinct indices chosen from $\{1, \ldots, N\} j(i, s) 1 \leqq i \leqq p, s=0$ or 1

$$
\sum_{s_{1} \ldots s_{p}=0,1}(-1)^{\sum s_{l}} v^{\Omega-\left\{j\left(1, s_{1}\right), \ldots, j\left(p, s_{p}\right)\right\}}=0 .
$$


Before proceeding to the proof we write down examples of (3.2.9) for $p=1,2$.

$$
\begin{array}{ll}
p=1: & v^{\Omega_{l}}=v^{\Omega_{j}} \quad \forall i, j \\
p=2: & v^{\Omega_{l}}+v^{\Omega_{k l}}=v^{\Omega_{i l}}+v^{\Omega_{k J}}
\end{array}
$$

where $\Omega_{r s}=\Omega-\{r\}-\{s\}$.

Proof. We consider the $\varrho$ component of (3.2.9). There are 3 cases.

1. $\varrho$ contains $j(i, 0)$ and $j(i, 1)$ for some $i, 1 \leqq i \leqq p$.

Then each term $v_{\varrho}^{\chi}$ in $(3.2 .9)_{\varrho}$ has $\chi \cap \varrho \neq \phi$ and vanishes.

2. $\varrho$ does not contain either $j(i, 0)$ or $j(i, 1)$ for some $i, 1 \leqq i \leqq p$.

Then two terms in the sum (3.2.9) $)_{e}$ which differ only in the value of $s_{i}$ are numerically equal but have opposite sign so the sum gives zero.

3. For each $i, 1 \leqq i \leqq p, \varrho$ contains just one of $j(i, 0), j(i, 1)$.

Then $|\varrho| \geqq p$ so each term in $(3.2 .9)_{\varrho}$ is zero by the remark preceding Lemma 3.2.2.

Lemma 3.2.11. There are at least $\left(\begin{array}{c}N \\ p\end{array}\right)-\left(\begin{array}{c}N \\ p-1\end{array}\right)$ independent relations in the set (3.2.9).

Proof. The relations (3.2.9) span a vector space $R(N, p)$; we must show $\operatorname{dim} R(N, p) \geqq\left(\begin{array}{c}N \\ p\end{array}\right)-\left(\begin{array}{c}N \\ p-1\end{array}\right)$. The proof is by induction on $N$; the proof is obvious for $N=2$ from (3.2.10). For general $N$ we define a map $f: R(N-1, p) \rightarrow R(N, p)$ by

$$
f\left[\Sigma a_{\chi} v^{\chi}\right]=\sum a_{\chi} v^{\chi \cup\{N\}}
$$

and a map $g: R(N, p) \rightarrow R(N-1, p-1)$ by

$$
g\left[\sum a_{\chi} v^{\chi}\right]=\sum_{\chi \ni N} a_{\chi} v^{\chi} .
$$

Then $f$ is an injection, $g$ is onto, and $g f=0$, so that

$$
\begin{aligned}
\operatorname{dim} R(N, p) & \geqq \operatorname{dim} R(N-1, p)+\operatorname{dim} R(N-1, p-1) \\
& \geqq\left(\begin{array}{c}
N-1 \\
p
\end{array}\right)-\left(\begin{array}{c}
N-1 \\
p-1
\end{array}\right)+\left(\begin{array}{c}
N-1 \\
p-1
\end{array}\right)-\left(\begin{array}{c}
N-1 \\
\mathrm{p}-2
\end{array}\right) \\
& =\left(\begin{array}{c}
N \\
p
\end{array}\right)-\left(\begin{array}{c}
N \\
p-1
\end{array}\right) .
\end{aligned}
$$

From Lemma 3.2.11 and the remark following Lemma 3.2.6 we see that the relations of Lemma 3.2.8 are a complete set of relations on the rows of $v$. This implies that the sequence

$$
0 \rightarrow R(N-1, p) \stackrel{f}{\rightarrow} R(N, p) \stackrel{g}{\rightarrow} R(N-1, p-1) \rightarrow 0
$$

is actually exact. 


\subsection{Dimension of $V_{N}$}

We now discuss the algebra $\mathscr{A}_{N}$ in the case in which the $\lambda_{i}$ have values corresponding to (2.1.1). Care must be taken in this specialization; in particular, the elements (2.4.29) (or more precisely their images under $\mathscr{L}$ ) no longer span $\mathscr{A}_{N}$. However, the formulae for the matrix representation

$$
\mathscr{L}(x) \psi_{\varrho}=\sum_{\chi \in \mathscr{M}} \mathscr{L}(x, \lambda)_{\chi \varrho} \psi_{\chi} \quad \varrho \in \mathscr{M}, x \in \mathbb{C}\left(\mathscr{G}_{N}\right)
$$

continue to hold when we specialize the values of the $\lambda_{i}$. (The matrix elements $\mathscr{L}(x, \lambda)_{\chi \varrho}$ are continuous in $\lambda$.) But note that the vectors $\left\{\psi_{\varrho}\right\}$ do not remain linearly independent when we specialize the $\lambda_{i}$. We obtain this reduction in the dimension of $V_{N}$ by application of the separation principle (Lemma 3.3.3).

Suppose that $f$ is a homogeneous function of $n$ complex variables, of degree $d$, which satisfies

(1) $f$ is holomorphic in the universal covering of the complement of an algebraic variety

$$
L \subset \mathbb{C}^{n}
$$

(2) if $z_{0}$ is a point not in $L$, the germs of $f$ with center $z_{0}$ span a finite dimensional vector space $V\left(z_{0}\right)$,

(3) if $z_{1}$ is a regular point of $L, \ell(z)=0$ a local equation of $L$ and $U$ a neighbourhood of $z_{1}$ sufficiently small that the cut region

$$
U^{\prime}=\{(z): z \in U, \quad \arg \ell(z) \neq 0\}
$$

is simply connected, there is an integer $p$ such that for any branch $f^{\prime}$ of $f \mid U^{\prime},[\ell(z)]^{p} f^{\prime}(z)$ is bounded in $U^{\prime}$.

Remark. Conditions (1)-(3) are essentially the conditions defining a function of Nilsson class [14], except that we have imposed a weaker form of the growth condition (3).

Lemma 3.3.2. Under the above assumptions $f^{\prime}$ has a decomposition

$$
f^{\prime}(z)=\sum_{k, \varrho}[\ell(z)]^{e}[\log \ell(z)]^{k} A_{k, \varrho}(z) .
$$

In (3.3.2) the summation is over a finite set of pairs $(k, \varrho), k$ a nonnegative integer and $\varrho$ a complex number and the functions $A_{k, \varrho}(z)$ are holomorphic in $U$.

Proof. $\pi_{1}(U-L)$ is infinite cyclic with generator $\alpha$ (say) and acts on the vector space $V\left(z_{2}\right)$ where $z_{2}$ is a base point in $U-L$. (3.3.2) is obtained by reducing the matrix $\mathscr{L}(\alpha)$ to Jordan canonical form (cf. [1]).

As in $\S 2.1$ we may consider the representation $\mathscr{L}$ of $\pi_{1}\left(\mathbb{C}^{n}-L ; z_{0}\right)$ defined on $V\left(z_{0}\right)$ by analytic continuation. 
Lemma 3.3.3 (separation principle). If in addition to conditions (1)-(3) formulated above, the homogeneous function $f$ satisfies

(4) no exponent $\varrho$ in any local decomposition (3.3.2) of a branch of $f$ about a regular point of $L$ is a negative integer, then the relation

$$
\mathscr{L}(\alpha) \Phi=\Phi \quad \forall \alpha \in \pi_{1}\left(\mathbb{C}^{n}-L ; z_{0}\right)
$$

implies that the function $\Phi \in V\left(z_{0}\right)$ is either zero, if $d$ is not a non-negative integer, or a homogeneous polynomial of degree $d$, if $d$ is a non-negative integer.

Proof. $\Phi$ evidently defines a single valued function on $\mathbb{C}^{n}-L$. Lemma 3.3.3 then follows from the growth condition (3) which forces $\Phi(z)$ to be rational and (4) which rules out the possibility of polar singularities on $L$.

We have already remarked that our integral (2.1.18) satisfies (1) and (2). When the $\lambda$ 's are specialized, conditions (3) and (4) follow from the Picard-Lefschetz theorem.

Lemma 3.3.4. If $\sum_{\chi \in \mathscr{M}} d_{\chi} v^{\chi}=0$ is a linear relation on the row vectors of the matrix $v$ of $\S 3.2$, the corresponding relation $\sum_{\chi \in \mathscr{M}} d_{\chi} \psi_{\chi}=0$ holds in $V_{N}$.

Proof. It is sufficient to consider the case in which the linear relation is one of those obtained in Lemma 3.2.8. From the separation principle it is enough to show

$$
a\left(\sum_{\chi \in \mathscr{M}} d_{\chi} \psi_{\chi}\right)=0, \quad b_{i}\left(\sum_{\chi \in \mathscr{M}} d_{\chi} \psi_{\chi}\right)=0 \quad 1 \leqq i \leqq N
$$

These relations may be directly verified using the explicit form of the coefficients $d_{\chi}$ corresponding to (3.2.9).

We now show that there are no more relations among the vectors $\Psi_{\chi}$. Let $W$ denote the vector space $\mathbb{C}^{2^{N-1}}$, and let $W^{\prime}$ be the subspace

$$
W^{\prime}=\left\{d_{\chi} \mid \sum_{\chi \in \mathscr{M}} d_{\chi} v^{\chi}=0\right\}
$$

Then the vectors $v^{\chi}$ span the dual space $\left(W / W^{\prime}\right)^{*} \subset W^{*}$. Let $f: W \rightarrow V_{N}$ be defined by

$$
f\left(\left\{d_{\chi}\right\}\right)=\sum_{\chi \in \mathscr{M}} d_{\chi} \Psi_{\chi} .
$$

$f$ is onto. Then (2.4.24) becomes

$$
\mathscr{L}[b(\psi) a b(\chi)] f(w)=\left(v^{\chi} \cdot w\right) f\left(u^{\psi}\right) .
$$

Lemma 3.3.6. The relations of Lemma 3.3.4 exhaust the relations among the vectors $\Psi_{\varrho}$. 
Proof. This is precisely the statement that the kernel of $f$ is $W^{\prime}$ (we know $\operatorname{ker} f \supset W^{\prime}$ by Lemma 3.3.4). Suppose then $w \in \operatorname{ker} f$, but $w \notin W^{\prime}$. Since the $v^{\chi}$ s $\operatorname{span}\left(W / W^{\prime}\right)^{*}$, there is some $\varrho \in \mathscr{M}$ with $\left(v^{o} \cdot w\right) \neq 0$; applying (3.3.5) with $\chi=\varrho, \psi=\Omega_{i j}$ gives

so that

$$
0=\left(v^{\varrho} \cdot w\right) f\left(u^{\Omega_{\imath \jmath}}\right)
$$

$$
f\left(u^{\Omega_{\imath j}}\right)=\left[\psi_{\Omega_{i}}+\psi_{\Omega_{j}}\right]=0
$$

But again from (3.3.5) (with $\psi=\phi$ )

$$
\mathscr{L}(a) \Psi_{\Omega_{l}}=f\left(u^{\phi}\right)=\mathscr{L}(a) \Psi .
$$

Thus $\mathscr{L}(a) \Psi=0$, contradicting (2.3.22).

Corollary 3.3.7. The dimension of $V_{N}$ is $2^{N}-\left(\begin{array}{c}N \\ {[N / 2]}\end{array}\right)$.

Proof. This follows from Lemmas (3.3.4) and (3.3.7), and Eq. (3.2.7).

As we have seen in $\S 2$ the representation $\mathscr{L}(\lambda)$ of $\mathscr{G}$ is irreducible for generic $\lambda$. However, in the specialized case under discussion the representation is reducible. The reducibility of the representation may be anticipated by considering the integral (2.1.0). Each discontinuity of $I(s)$ is given by integrating the differential form which appears in (2.1.0) over a suitable cycle. The cycles which arise in this way are of a particular form - they are coboundaries of cycles which lie on the intersection of one or more of the pole varieties

$$
S_{i}=\left\{(k): k_{i}^{2}+s_{i}=0\right\} .
$$

The Leray residue calculus may thus be used to express the discontinuities as integrals in which one or more of the propagator poles $\left(k_{i}^{2}+s_{i}\right)^{-1}$ in (2.1.0) is replaced by a $\delta$-function [8]. We may define a sequence

$$
V_{N}=W^{0} \supset W^{1} \supset \cdots \supset W^{N} \supset \phi
$$

of subspaces of $V_{N}$ :

$W^{k}=\left\{\mathscr{I} \in V_{N}: \mathscr{I}\right.$ has a representation as a linear combination of discontinuities, each one of which can be written as an integral in momentum space in which $k$ of the propagator poles are replaced by $\delta$-functions $\}$. The subspaces $W^{k}$ of (3.3.8) are invariant under $\mathscr{L}$ so $\mathscr{L}$ is reducible.

We now define (from a purely algebraic point of view) a sequence $V^{k}$ of subspaces of $V_{N}$, which are invariant under $\mathscr{L}$. Presumably this sequence can be identified with the sequence (3.3.8).

Lemma 3.3.9. There is a unique minimal subspace of $V_{N}$ invariant under $\mathscr{L}$. 
Proof. Let $V$ be an invariant subspace of $V_{N}$, and $x$ a non-zero vector in $V$. Then we may find a vector $v^{x}$ such that $v^{x} \cdot f^{-1}(x) \neq 0$ (where $f: W \rightarrow V_{N}$ is defined above Lemma 3.3.6), and hence

$$
\mathscr{L}[b(\psi) a b(\chi)] x=\left[v^{\chi} \cdot f^{-1}(x)\right] f\left(u^{\psi}\right) \neq 0
$$

for all $\psi \in \mathscr{M}$. $V$ thus contains all the vectors $f\left(u^{\psi}\right)$ and hence their span. It is easy to check that this subspace $V_{\min }$ of $V$ is invariant.

For each $\chi \in \mathscr{M}$ we can write

where

$$
\begin{aligned}
\mathscr{L}(a(\chi)) \psi_{\varrho} & =\mathscr{L}\left(\beta(\gamma) a \beta(\chi)^{-1}\right) \psi_{\varrho} \\
& =\ell_{\varrho}^{\chi}\left(\sum m_{\mu}^{\chi} \psi_{\mu}\right),
\end{aligned}
$$

and

$$
\ell^{x}=\sum_{\psi \subset x}(-1)^{|x|} v^{\psi}
$$

$$
m^{\chi}=\sum_{\psi \subset \chi} u^{\psi}
$$

From (3.3.13) it follows that $V_{\min }=\operatorname{span}\left\{f\left(m^{\chi}\right), \chi \in \mathscr{M}\right\}$, i.e. the span of the discontinuities for the leading Landau variety. Thus certainly

$$
V_{\min } \subset W^{N}
$$

which motivates the definition $V^{N}=V_{\min }$. For $k, 0 \leqq k \leqq N-1$ we define $V^{k}$ to be the minimal invariant subspace of $V_{N}$ containing all vectors $\psi_{\chi}$ with $|\chi|=k$. Clearly $V^{k} C W^{k}$.

Lemma 3.3.14. For

$$
k \geqq\left[\frac{N+1}{2}\right] \quad V^{k}=V_{\min } .
$$

For

$$
\begin{gathered}
k<\left[\frac{N+1}{2}\right] \operatorname{dim}\left(V^{k}-V^{k+1}\right)=\left(\begin{array}{l}
N \\
k
\end{array}\right)-\left(\begin{array}{c}
N \\
k-1
\end{array}\right) . \\
\operatorname{dim} V_{\min }=2^{N}-\left(\begin{array}{c}
N \\
{[(N+1) / 2]}
\end{array}\right)-\left(\begin{array}{c}
N \\
{[(N+1) / 2]}
\end{array}\right) .
\end{gathered}
$$

This Lemma is readily proved with the help of Lemma 3.2.6.

On $W^{N}$, and hence on $V_{\min } \subset W^{N}$, there is a natural scalar product defined by

$g_{1} \cdot g_{2}=$ Kronecker index of a pair of cycles $\underline{g}_{1}, \underline{g}_{2}$ on

$S_{1} \cap \cdots \cap S_{N}$ defining the discontinuities $g_{1}, g_{2}$ of $I(s)$. 
The scalar product is symmetric for $N$ even, antisymmetric for $N$ odd. This scalar product on $V_{\min }$ may be recovered from our algebraic results. We may regard the scalar product as defining a linear map $\tau$ of $V_{\min }$ into its dual space. From the Picard-Lefschetz theorem we know that for each $\chi \in \mathscr{M}$

$$
\tau\left(f\left(m^{x}\right)\right)=c_{\chi} \ell^{x},
$$

where $\ell^{x}$ is to be regarded as defining an element of $V_{\min }^{*}$ via

$$
\ell^{x} \cdot x=\ell^{x} \cdot f^{-1}(x) \quad x \in V_{\min }
$$

and $c_{\chi}$ is some constant to be determined. The symmetry property of the scalar product noted above gives

$$
\tau\left(f\left(m^{\chi_{1}}\right)\right) \cdot f\left(m^{\chi_{2}}\right)=(-1)^{N} \tau\left(f\left(m^{\chi_{2}}\right)\right) \cdot f\left(m^{\chi_{1}}\right) .
$$

But a direct computation gives

$$
\ell^{x_{1}} \cdot m^{x_{2}}=(-1)^{N} \ell^{x_{2}} \cdot m^{x_{1}}
$$

so if we normalize $\tau$ by taking $c_{\phi}=1$ we have $c_{\chi}=1$ for all $\chi$. If for some constants $a_{x}$

$$
\sum a_{\chi} f\left(m^{\chi}\right)=0
$$

(3.3.16) shows that for any $\psi$

$$
\begin{aligned}
\Sigma a_{\chi} \ell^{\chi} \cdot f\left(m^{\psi}\right) & =\Sigma a_{\chi} \ell^{\chi} \cdot m^{\psi} \\
& =(-1)^{N} \Sigma a_{\chi} \ell^{\psi} \cdot m^{\chi}=0,
\end{aligned}
$$

i.e. that $\sum a_{x} \ell^{x}$ defines the element $0 \in V_{\min }^{*}$. The map $\tau$ defined by (3.3.15) with $c_{\chi}=1$ may thus be extended to a linear map $\tau: V_{\min } \rightarrow V_{\min }^{*}$ as required by homological considerations.

\subsection{Equal Mass Case}

We now consider the integrals (2.1.18) and (2.1.1) in the case where all internal lines have the same mass:

$$
s^{\prime}=s_{1}=s_{2}=\cdots=s_{N} .
$$

Let $\mathbb{C}^{2}$ denote the subspace of $\mathbb{C}^{N+1}$ specified by (3.4.1); we thus wish to find the representation $\mathscr{L}_{0}$ of $\pi_{1}\left(\mathbb{C}^{2}-\mathbb{C}^{2} \cap L\right)$ generated by $I(s, \lambda) \mid \mathbb{C}^{2}$ [see (2.1.18)] or $I(s) \mid \mathbb{C}^{2}$ [see (2.1.1)]. Our base point $B$ for $\pi_{1}\left(\mathbb{C}^{N+1}-L\right)$ $=\mathscr{G}_{N}$ was chosen to lie in $\mathbb{C}^{2}$; thus if we also use $B$ as a base point for $\pi_{1}\left(\mathbb{C}^{2}-\mathbb{C}^{2} \cap L\right)$ there is a natural map

We clearly have

$$
e: \pi_{1}\left(\mathbb{C}^{2}-\mathbb{C}^{2} \cap L\right) \rightarrow \mathscr{G}_{N} .
$$

$$
\mathscr{L}_{0}=\mathscr{L} e,
$$


so that finding the representation $\mathscr{L}_{0}$ is equivalent to finding $e$. Note that even if our sole objective were to construct the monodromy group in the equal mass case, it would still be useful to consider the case of unequal masses. For the fact that the Feynman integral regarded as a function in $s, s^{\prime}$ is a restriction of a function analytic in $s, s_{i} 1 \leqq i \leqq N$ gives us additional information on its monodromy ring which is expressed by the factorization of $\mathscr{L}_{0}$ through $e$.

Now $\mathbb{C}^{2} \cap L$ is given by

$$
\begin{aligned}
& s^{\prime}=0 \\
& s_{0}=k^{2} s^{\prime} \quad(k=N, N-2, \ldots ; k \geqq 0) \\
& s_{0}=0 .
\end{aligned}
$$

We let $\alpha_{k}(k>0)$ denote the generator of $\pi_{1}\left(\mathbb{C}^{2}-\mathbb{C}^{2} \cap L\right)$ corresponding to the line $s_{0}=k^{2} s^{\prime}$ (see $\S 2.2$ ), and $\beta$ denote the generator for $s^{\prime}=0$; we do not need to consider the generator for $s_{0}=0$ since its image under $\mathscr{L}_{0}$ may be obtained from $\beta$, the $\alpha_{k}$, and the loop at infinity (which is again represented by $c_{\infty}$ times the identity). The line $s_{0}=k^{2} s^{\prime}(k>0)$ is the intersection of all surfaces $L_{N+1}(\chi)(2.2 .3)$ with $|\chi|=\frac{N-k}{2}$. Since the generators $\alpha(\chi)$ for these surfaces commute, we have

$$
e\left(\alpha_{k}\right)=\prod_{|\chi|=(N-k) / 2} \alpha(\chi) .
$$

Similarly,

$$
e(\beta)=\beta_{1} \ldots \beta_{N} .
$$

We now turn to the question of the dimension of the representation $\mathscr{L}_{0}$, that is, the dimension of the subspace $V_{N}^{0}$ of $V_{N}$ spanned by vectors

$$
\left\{\mathscr{L}_{0}(\gamma) \Psi \mid \gamma \in \pi_{1}\left(\mathbb{C}^{2}-\mathbb{C}^{2} \cap L\right)\right\} .
$$

Theorem 3.4.4. a) For generic $\lambda, V_{N}^{0}=V_{N}$, so that $\operatorname{dim} V_{N}^{0}=2^{N}-1$. b) For the $\lambda$ 's specialized to correspond to (2.1.1), $V_{N}^{0}$ is spanned by the linearly independent vectors

$$
\Psi_{k}=\sum_{|\varrho|=k} \Psi_{\varrho} \quad(k=0,1, \ldots N-1)
$$

so that $\operatorname{dim} V_{N}^{0}=N$.

We remark that the conclusion of b) is obtained if the $\lambda$ 's are only specialized to satisfy

$$
\lambda_{1}=\lambda_{2}=\cdots=\lambda_{N} .
$$


Proof. a) We will show that the orbit of $\Psi$ under the action of $\beta$ spans $V_{N}$. For $n \geqq 1$,

where

$$
\mathscr{L}_{0}\left(\beta^{n}\right) \Psi=\sum_{\varrho \in \mathcal{M}} A(\varrho, n) \Psi_{\varrho}
$$

$$
A(\varrho, n)=\prod_{i \in \varrho}\left[\frac{\left(1+B_{i}\right)^{n}-1}{B_{i}}\right]
$$

as may be easily verified by induction. Thus it suffices to prove that the matrix $\{A(\varrho, n)\}\left(\varrho \in \mathscr{M}, n=1, \ldots, 2^{N}-1\right)$ is nonsingular. But $A(\varrho, n)$ is equivalent to the matrix

$$
\begin{aligned}
A^{\prime}(\varrho, n) & =\sum_{\chi<\varrho}\left(\prod_{\chi} B_{i}\right) A(\chi, n) \\
& =\left[\prod_{\varrho}\left(1+B_{\imath}\right)\right]^{n}
\end{aligned}
$$

The determinant of $A^{\prime}$ is a Vandermonde determinant and is non-zero as long as

$$
\prod_{\varrho}\left(1+B_{i}\right) \neq \prod_{\varrho^{\prime}}\left(1+B_{i}\right)
$$

for any distinct $\varrho$ and $\varrho^{\prime}$ in $\mathscr{M}$.

b) In this case

$$
B_{1}=B_{2}=\cdots=B_{N}=0
$$

so that (3.4.6) becomes

The matrix

$$
\mathscr{L}_{0}\left(\beta^{n}\right) \Psi=\sum_{k=0}^{n=1} n^{k} \Psi_{k} \quad(n \geqq 1) .
$$

$$
\left\{n^{k}\right\} \quad(n=1, \ldots N ; k=0, \ldots N-1)
$$

has non-zero determinant, so the vectors $\Psi_{k}$ all lie in $V_{N}^{0}$. It follows from Lemma 3.3.6 that these vectors are linearly independent. Finally, (3.4.7) implies that the operators $\mathscr{L}(\beta)$ and $\mathscr{L}\left(\alpha_{k}\right)$ are completely symmetric in the indices $1, \ldots N$. The vector space spanned by the $\Psi_{k}$ is therefore invariant under these operators, and must coincide with $V_{N}^{0}$.

\subsection{The Zero Mass Case}

We now study the representations generated when the mass of one line in the graph $G_{N}$ is set equal to zero. Since the amplitude $I(s, \lambda)$ is singular on the surface $\mathbb{C}^{N}=\left\{s \mid s_{N}=0\right\}$, some care is needed in this discussion. For generic $\lambda$, the Picard-Lefschetz theorem implies that in a neighbourhood of any point of $\mathbb{C}^{N}-\mathbb{C}^{N} \cap L$ we may write

$$
I(s, \lambda)=s_{N}^{-\left(1+\lambda_{N}\right)} R(s, \lambda)+S(s, \lambda),
$$


where $R$ and $S$ are analytic in this neighbourhood. It is natural to study the analytic properties of both $R(s, \lambda) \mid \mathbb{C}^{N}$, a sort of residue of $I(s, \lambda)$, and $S(s, \lambda) \mid \mathbb{C}^{N}$, which is indeed the restriction of $I(s, \lambda)$ to $\mathbb{C}^{N}$ whenever $\operatorname{Re} \lambda_{N}<-1$.

Recall that $\Psi$ is the germ of the physical sheet of $I(s, \lambda)$ defined at the base point $B=(1, \varepsilon, \ldots \varepsilon)$. From (3.5.1) we see that the formulae

$$
\begin{aligned}
& \Phi=\lim _{s_{N} \rightarrow 0} s_{N}^{\left(1+\lambda_{N}\right)} \frac{b_{N}}{B_{N}} \Psi, \\
& \Theta=\lim _{s_{N} \rightarrow 0}\left(1-\frac{b_{N}}{B_{N}}\right) \Psi
\end{aligned}
$$

give well defined functions at the base point $B=(1, \varepsilon, \ldots \varepsilon, 0)$ of $\mathbb{C}^{N}-\mathbb{C}^{N} \cap L$, which may be analytically continued throughout $\mathbb{C}^{N}-\mathbb{C}^{N} \cap L$. $\Phi$ and $\Theta$ generate representations

respectively.

$$
\begin{aligned}
& \mathscr{L}_{1}: \mathbb{C}\left(\mathscr{G}_{N-1}\right) \rightarrow L\left(V_{N}^{(1)}\right), \\
& \mathscr{L}_{2}: \mathbb{C}\left(\mathscr{G}_{N-1}\right) \rightarrow L\left(V_{N}^{(2)}\right),
\end{aligned}
$$

Theorem 3.5.4. There are natural isomorphisms

and for $i=1,2$, and $y \in \mathscr{G}_{N-1}$,

$$
\begin{aligned}
& f_{1}: V_{N}^{(1)} \rightarrow b_{N} V_{N}, \\
& f_{2}: V_{N}^{(2)} \rightarrow\left(1-\frac{b_{N}}{B_{N}}\right) V_{N},
\end{aligned}
$$

$$
\mathscr{L}_{i}(y)=f_{i}^{-1}\{\mathscr{L} j(y)\} f_{i} .
$$

Here $j: \mathscr{G}_{N-1} \rightarrow \mathscr{G}_{N}$ is the map of $\S 2.2$.

Proof. For $y \in \mathscr{G}_{N-1}$ we define

$$
\begin{aligned}
& f_{1}\left[\mathscr{L}_{1}(y) \Phi\right]=\left[\mathscr{L}_{j}\right](y) b_{N} \Psi, \\
& f_{2}\left[\mathscr{L}_{2}(y) \Theta\right]=\left[\mathscr{L}_{j}\right](y)\left[1-\frac{b_{N}}{B_{N}}\right] \Psi .
\end{aligned}
$$

The above properties are then easily verified, using the fact that $b_{N}$ commutes with $j\left(\mathscr{G}_{N-1}\right)$. Note that (3.5.5) states essentially that $\mathscr{L}_{i}$ is given by restricting $\mathscr{L}$ to $b_{N} V_{N}$ or $\left(1-\frac{b_{N}}{B_{N}}\right) V_{N}$, for $i=1,2$ respectively.

We will not discuss these representations in detail, but will point out several immediate consequences of Theorem 3.5.4. Define

$$
\begin{aligned}
& \Phi^{\varrho}=\mathscr{L}_{1}\left(\prod_{\varrho} b_{i}^{\prime}\right) \Phi \quad\left(\varrho \subset \Omega_{N}\right), \\
& \Theta_{\varrho}=\mathscr{L}_{2}\left(\prod_{\varrho} b_{i}^{\prime}\right) \Theta
\end{aligned}
$$


where again we have used primes to denote elements of $\mathbb{C}\left(\mathscr{G}_{N-1}\right)$. The vector space $V_{N}^{(1)}$ has dimension $2^{N-1}-1$, and we may take as basis $\left\{\Phi_{\varrho} \mid \varrho \neq \Omega_{N}\right\}$ (note $\Phi_{\Omega_{N}}=0$ ). In fact, it follows from Lemma 2.4.28 that $\mathscr{L}_{1}$ is isomorphic to the standard representation associated with the graph $G_{N-1}$. The vector space $V_{N}^{(2)}$ has dimension $2^{N-1}$ with basis $\left\{\Theta_{\varrho}\right\}$. It may be shown that $\mathscr{L}_{i}\left[\mathbb{C}\left(\mathscr{G}_{N-1}\right)\right]$ is a complete matrix ring for $i=1,2$, of dimension $2^{N-1}-1$ and $2^{N-1}$, respectively.

We will not discuss the modifications of this behavior which occur when the $\lambda$ 's are specialized to correspond to (2.1.1). Such a discussion could easily be obtained by the techniques of this section, $\S 3.3$, and $\S 4$.

\section{§ 4. The Renormalized Integrals}

\subsection{Introduction}

In this section we discuss the analytic structure of the renormalized integral in the case in which the dimension $m$ of space-time is set equal to 4. A similar discussion could be given for other values of $m$.

To define the renormalized integral for the graph $G_{N}$ we use the method of analytic renormalization developed in [15]. We denote by $\mathscr{I}=\left\{\mathscr{I}_{N}\right\}$ an arbitrary generalized evaluator in the sense of $[15]^{5}$, and by $\mathscr{I}^{0}$ the particular evaluator defined by

$$
\mathscr{I}_{N}^{0} F(\mu)=\sum_{\sigma \in S^{N}} \int_{\left|\mu_{1}\right|=R_{\sigma(1)}} \ldots \int_{\left|\mu_{N}\right|=R_{\sigma(N)}} \frac{F(\mu) d \mu_{1} \ldots d \mu_{N}}{\mu_{1} \ldots \mu_{N}} .
$$

In (4.1.1) $F(\mu)$ is a function such that for some integer $m$

$$
F(\mu)\left(\prod_{\chi \in \mathcal{M} \cup\{\Omega\}}\left(\sum_{i \in \chi} \mu_{i}\right)\right)^{m}
$$

is holomorphic in the neighbourhood of $\mu=0$. The summation is over all permutations of $1, \ldots, N$ and $R_{1}, \ldots, R_{N}$ are small positive real numbers satisfying

$$
R_{i}>R_{1}+\cdots+R_{i-1} \quad \text { for } \quad 2 \leqq i \leqq N .
$$

The integral (2.1.18) is convergent for

$$
\sum_{i=0}^{N} \operatorname{Re} \lambda_{i}+(N+2)>0
$$

\footnotetext{
${ }^{5}$ The evaluator $\mathscr{I}$ must satisfy the following additional condition. Let $F(\mu)$ be a function as in (4.1.1), and let $F_{z}\left(\mu_{1}, \ldots, \mu_{N}\right)=F\left(z \mu_{1}, \ldots, z \mu_{N}\right)$, for any $z \in \mathbb{C}$. Then $\mathscr{I} F_{z}=\mathscr{I} F$.
} 
and defines for $s$ in the neighbourhood of the base point $B$ a function holomorphic in $\lambda$ in this region. However, the value of $\lambda$ corresponding to $m=4$

$$
\lambda_{0}=0 \quad \lambda_{i}=-2 \quad 1 \leqq i \leqq N
$$

does not lie in the region of convergence. In [15] the function

$$
J(s, \lambda)=\left.I(s, \lambda)\right|_{\lambda_{0}=0}
$$

is considered, and it is shown that for $s$ in the neighbourhood of $B$ this function is the restriction to the region

$$
\sum_{i=1}^{N} \operatorname{Re} \lambda_{i}+(N+2)>0
$$

of a function $E(s, \lambda)$ meromorphic in the entire space $\mathbb{C}^{N}$ of $\lambda$. We will need the following more precise statement about the behaviour of $E(s, \lambda)$ in the neighbourhood of the point (4.1.5): if $\mu_{i}=2 \pi i\left(\lambda_{i}+2\right)$, $E(s, \lambda)$ admits a representation

$$
E(s, \lambda)=\sum_{\sigma \in A^{N}} \frac{E_{\sigma}(s, \mu)}{\left(\mu_{\sigma(1)}+\cdots+\mu_{\sigma(N)}\right) \ldots\left(\mu_{\sigma(1)}+\mu_{\sigma(2)}\right)} .
$$

Here the summation is over all even permutations of $\{1, \ldots, N\}$ and $E_{\sigma}(s, \mu)$ is holomorphic in $\mu$ in the neighbourhood of $\mu=0$. The physical sheet of the renormalized amplitude is then defined to be

$$
\mathscr{I} E(s, \lambda)=F(s) .
$$

The extension construction can be applied also to the integrals which define other sheets of $J(s, \lambda)$. If $\gamma \in \mathscr{G}_{N}$ we obtain in this way the commutative diagram

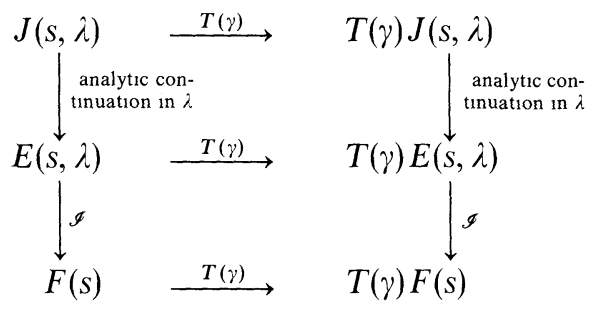

$T(\gamma)$ denotes the operation of analytic continuation along some representative loop for $\gamma$. As in $\S 2.1$ we may introduce the vector space $V_{N, \mathscr{G}}$ spanned by germs of $F_{N}(s)$ with center $B$ and consider the representation

$$
\mathscr{L}_{\mathscr{I}}: \mathscr{G}_{N} \rightarrow L\left(V_{N, \mathscr{I}}\right)=G L(d, \mathbb{C}) \quad d=\operatorname{dim} V_{N, \mathscr{I}}
$$


defined by analytic continuation. (We show below that $\operatorname{dim} V_{N, \mathscr{I}}$ is finite.)

To determine $\mathscr{L}_{\mathscr{g}}$ we start from the results established in $\S 2$ for the generic case, which according to the upper square of (4.1.10) are valid also for generic $\lambda$ in the neighbourhood of $\mu=0$. The vectors $\psi_{\chi} \chi \in \mathscr{M}$ span $V_{N}(\lambda)$, but it does not follow that the vectors $\mathscr{I} \psi_{\chi} \chi \in \mathscr{M} \operatorname{span} V_{N, \mathscr{I}}$. For when we express an analytic continuation of $E(s, \lambda)$ along some path as a linear combination of $\psi_{\chi}$ the coefficients $c_{\chi}$ depend on the parameters $\lambda$ and

$$
\mathscr{I}\left(c_{\chi}(\mu) \psi_{\chi}\right) \text { may not be equal to } c_{\chi}(0) \mathscr{I} \psi_{\chi} \text {. }
$$

A simple example should make this point clear and illustrate the technique of constructing $\mathscr{L}_{\mathscr{g}}$. Consider the function

$$
\frac{z^{\mu}}{\mu} \text {. }
$$

For $\mu \neq 0$ this function is holomorphic in $\mathbb{C}-\{0\}$. Its germs over a nonsingular point span a one-dimensional space on which the infinite cyclic group $\pi_{1}(\mathbb{C}-\{0\})$ acts. If $\gamma$ denotes the generator of this group the representation is given by

$$
\mathscr{L}(\gamma) \psi=(\exp (2 \pi i \mu)) \psi .
$$

The operation $\mathscr{I}$ applied to (4.1.11) gives

$$
\log z \text {. }
$$

The germs of this function span a vector space of dimension 2, with basis

$$
\psi_{1}=\mathscr{I} \psi=\log z \quad \text { and } \quad \psi_{2}=\mathscr{I}(\mu \psi)=1 .
$$

The action of $\pi_{1}(\mathbb{C}-\{0\})$ on $\psi_{1}, \psi_{2}$ may be deduced from (4.1.12)

$$
\begin{aligned}
\mathscr{L}_{\mathscr{I}}(\gamma) \psi_{1} & =\mathscr{I}(\mathscr{L}(\gamma) \psi)=\mathscr{I}(\exp (2 \pi i \mu) \psi) \\
& =\psi_{1}+2 \pi i \psi_{2}, \\
\mathscr{L}_{\mathscr{I}}(\gamma) \psi_{2} & =\mathscr{I}(\mu \mathscr{L}(\gamma) \psi)=\mathscr{I}(\mu \exp (2 \pi i \mu) \psi) \\
& =\psi_{2}
\end{aligned}
$$

since $\mathscr{I}\left(\mu^{k} \psi\right)=0$ for $k \geqq 2$.

Returning to the discussion of the Feynman amplitudes, we denote by $M(\chi)$ the set of all monomials

$$
\mu^{m}=\prod_{i \in \chi} \mu_{i}^{m_{1}}
$$


in the variables $\left\{\mu_{i} i \in \chi\right\}$. Here $m_{i}, i \in \chi$ are non-negative integers and we use the customary multi-index notation $m=\left(m_{i}, i \in \chi\right)$

Set

$$
|m|=\sum m_{i} \quad m !=\prod_{i} m_{i} !
$$

$$
A(\chi, m)=\mathscr{I}\left(\mu^{m} \psi_{\chi}\right) .
$$

From our results for the generic case it is clear that these vectors span $V_{\mathscr{I}, N}$ - if we take our expression for $\mathscr{L}(a), \mathscr{L}\left(b_{i}\right)$ applied to $\psi_{\chi}$ as a sum of vectors $\Sigma c_{e}(\mu) \psi_{\mathscr{C}}$, multiply by $\mu^{m}$ and apply $\mathscr{I}$ we obtain corresponding expressions for $\mathscr{L}_{\mathscr{I}}(a), \mathscr{L}_{\mathscr{I}}\left(b_{i}\right)$ applied to $A(\chi, m)$ as a sum of vectors $A\left(\chi^{\prime}, m^{\prime}\right)$. (The explicit formulae are given in $\S 4$.2.) $V_{\mathscr{I}, N}$ has finite dimension in view of Lemma 4.1.18.

Lemma 4.1.18. $A(\chi, m)=0$ unless

i) $|\chi|+|m|<N$,

ii) $m \in M\left(\chi^{\prime}\right)$.

Proof. For $\chi=\phi$ this follows immediately from (4.1.8). For $\chi \neq \phi$ we need a corresponding decomposition of $\psi_{\chi}$. In momentum space $\psi_{\chi}$ may be written as a repeated integral over the momentum vectors $k_{i}$ $i \in \chi^{\prime}$ and then over the $k_{i} i \in \chi$. The first integration gives the physical sheet of the graph $G_{N-|\chi|}$ and the second integration is over a compact region. If we substitute for the first integral its decomposition (4.1.8) we obtain for $\psi_{\chi}$ the decomposition

$$
\psi_{\chi}(s, \mu)=\sum_{\sigma \in A^{N-|x|}} \frac{E_{\varrho}^{\chi}(s, \mu)}{\left(\mu_{\sigma(1)}+\cdots+\mu_{\sigma(N-|\chi|)}\right) \ldots\left(\mu_{\sigma(1)}+\mu_{\sigma(2)}\right)},
$$

where the summation is over even permutations of the indices in $\chi^{\prime}$. The statements of Lemma 4.1.18 follow immediately from 4.1.19.

The only essential point in the determination of $\mathscr{L}_{\mathscr{I}}$ remaining is to decide what linear relations hold between the vectors $A(\chi, m)$. We exhibit in $\S 4.2$ a number of these linear relations but we are not able by our algebraic methods to determine the dimension of $V_{N, \mathscr{I}}$ because there are a number of vectors in $V_{N, \mathscr{S}}$ representing single valued functions and we cannot decide how many of these may be zero.

A comparison between the method of analytic renormalization and the method of subtraction yields the following

Lemma 4.1.20. For $|m| \geqq 1 A(\chi, m)$ is a polynomial in $s_{0}$.

Proof. For $\chi=\phi$ we note that the proof of the equivalent of the method of subtraction and that of analytic renormalization gives

$$
\psi=E\left(s_{0}, \lambda\right)=\left.\sum_{j=0}^{\omega(G)} \frac{s_{0}^{j}}{j !}\left(\frac{d^{j}}{d s_{0}^{j}} E(s, \lambda)\right)\right|_{s_{0}=0}+\mathscr{R} E(s, \lambda),
$$


where $\mathscr{R} E(s, \lambda)$ is holomorphic in $\lambda$ for $\mu=0$. Multiplying (4.1.21) by $\mu^{m}$ and applying $\mathscr{I}$ we obtain

$$
A(\phi, m)=\sum_{j=0}^{\omega(G)} \frac{s_{0}^{j}}{j !}\left(\left.\left(\frac{d^{j}}{d s_{0}^{j}} E(s, \lambda)\right)\right|_{s_{0}=0}\right)
$$

a polynomial in $s_{0}$ of degree $\leqq \omega(G)$.

The proof for $\chi \neq \phi$ is reduced to the preceding case by the argument used in the proof of Lemma 4.1.18.

We will obtain more precise information on the functional nature of the $A(\chi, m)$ in $\S 4.3$.

\subsection{The Linear Relations on the $A(\chi, m)$}

Lemma 4.2.1. For all $\psi \in \mathscr{M}$ and multi-indices $m, 0<|m|<N-1-|\psi|$ we have

$$
\begin{aligned}
& \sum_{\substack{m^{\prime} \in M\left(\psi^{\prime}\right) \\
1 \leqq\left|m^{\prime}\right| \leqq N-1-|\psi|-|m|}}(-1)^{\left|m^{\prime}\right|} \frac{1}{m^{\prime} !} A\left(\psi, m+m^{\prime}\right) \\
& \quad+\sum_{\eta \supsetneq \psi} \sum_{\substack{m^{\prime} \in M\left(\eta^{\prime}\right) \\
0 \leqq\left|m^{\prime}\right| \leqq N-1-|\eta|-|m|}}(-1)^{\left|m^{\prime}\right|+|\eta-\psi|} \frac{1}{m^{\prime} !} A\left(\eta, m+m^{\prime}\right)=0 .
\end{aligned}
$$

Proof.For all $\psi \in \mathscr{M}$ the vector $\mathscr{L}(b(\psi) a) \Psi \in V_{N}(\lambda)$ can be represented as an integral over a compact contour in momentum space, or in $\alpha$-space over a contour which does not bound onto any $\alpha_{i}=0$ plane. It therefore has no divergences as $\mu \rightarrow 0$, i.e. it defines a function holomorphic in $\mu$ in the neighbourhood of $\mu=0$. Thus for all $m$ with $|m|>0$ we have from (2.4.24)

$$
\mathscr{I}\left\{\mu^{m} \sum_{\eta \in \mathscr{M}} u_{\eta}^{\psi} \psi_{\eta}\right\}=0 .
$$

Substituting the explicit form of the coefficients as functions of $\mu$ given by (2.4.26) and (2.3.11), (2.3.12) we obtain (4.2.2).

Examples. For $N=2$ Lemma 4.2 .1 gives us no relation.

For $N=3$ we obtain 3 relations $(m=\{i\}, \psi=\phi)$

$$
-\sum_{j \neq i} \mathscr{I}\left(\mu_{i} \mu_{j} \psi\right)=\sum_{j} \mathscr{I}\left(\mu_{i} \mu_{j} \psi\right),
$$

We have now expressed in the above Lemma the fact that discontinuities of $\Psi$ across the leading Landau singularity have no divergences, and in (4.1.29) the fact that discontinuities $\Psi_{\chi}$ across one or more of the internal mass singularities $L_{i}$ have only divergences corresponding to certain subgraphs of $G_{N}$. It remains to decide what divergences may appear in a discontinuity taken across the second-type singularity $L_{0}$. 
In the case $m=2 \mathscr{L}\left(b_{0}^{J}\right) V_{N} \subset V_{\min }$ the subspace spanned by discontinuities across the leading singularity. This does not mean, however, that the functions $\mathscr{L}\left(b_{0}^{j}\right) \psi$ have no divergences in the case $m=4$. In fact the pinch corresponding to the second-type singularity $s_{0}=0$ is given by

$$
d(\alpha)=0 \quad \sum_{i=1}^{N} \alpha_{i} s_{i}=0 .
$$

For $N=2,3$ this does not intersect the $\alpha_{i}=0$ planes so $\mathscr{L}\left(b_{0}^{j}\right) \psi$ has no divergences, i.e. is holomorphic in $\mu$ for $\mu=0$. But for $N>3$ it has nonzero intersection with the sets

$$
\left\{(\alpha): \alpha_{i}=0 \forall i \in \chi\right\}
$$

provided $|\chi| \leqq N-3 . \mathscr{L}\left(b_{0}^{j}\right) \psi$ thus has a corresponding decomposition

$$
\mathscr{L}\left(b_{0}^{j}\right) \psi=\Sigma \frac{H_{i_{1} \ldots i_{N-3}}(s, \mu)}{\left(\sum_{i \in \Omega_{i_{1}}} \mu_{i}\right) \ldots\left(\sum_{i \in \Omega_{i_{1} \ldots i_{N-3}}} \mu_{i}\right)},
$$

where the summation is over all ordered subsets containing $N-3$ indices, and the functions $H$ are holomorphic in $\mu$ at $\mu=0$. (4.2.7) can be rewritten in an equivalent form by using (2.4.5) and noting that all terms involving $a$ define functions holomorphic in $\mu$ and may be absorbed into the functions $H$. The resulting formula no longer contains $j$. A similar discussion may be given also for $\mathscr{L}\left(b_{0}^{j}\right) \psi_{\chi} \chi \neq \phi$.

We conclude this section by showing how the decomposition formulae (4.1.8), (4.1.29), (4.2.7) may be used to obtain further linear relations on the $A(\chi, m)$.

Consider for definiteness (4.1.8). We obtain a linear relation

$$
\sum c_{m} A(\phi, m)=0
$$

if we can find a polynomial

$$
\sum c_{m} \mu^{m}=P(\mu)
$$

of degree $N-1$ such that for all $\sigma \in A^{N}$

$$
\mathscr{I}\left(\frac{P(\mu)}{\left(\mu_{\sigma(1)}+\cdots+\mu_{\sigma(N)}\right) \cdots\left(\mu_{\sigma(1)}+\mu_{\sigma(2)}\right)}\right)=0 .
$$

For

$$
\begin{aligned}
& \mathscr{I}\left(\frac{P(\mu) E_{\sigma}(s, \mu)}{\left(\mu_{\sigma(1)}+\cdots+\mu_{\sigma(N)}\right) \ldots\left(\mu_{\sigma(1)}+\mu_{\sigma(2)}\right)}\right) \\
& =E_{\sigma}(s, 0) \mathscr{I}\left(\frac{P(\mu)}{\left(\mu_{\sigma(1)}+\cdots+\mu_{\sigma(N)}\right) \ldots\left(\mu_{\sigma(1)}+\mu_{\sigma(2)}\right)}\right) .
\end{aligned}
$$


(4.2.10) gives a set of linear equations on the coefficients $c_{m}$. A first sight there are too many equations at least for large $N$ and one would expect only trivial solutions. But by looking for solutions with some symmetry and exploiting the symmetry properties of $\mathscr{I}$ we can show that there are non-trivial solutions for any $N$ and any choice of $\mathscr{I}$. It appears difficult to determine how many solutions exist in general. Note that the coefficients in the linear equations for the $c_{m}$ depend on $\mathscr{I}$ so that to obtain the linear relations (4.2.8) explicitly in a particular case we must choose a particular $\mathscr{I}$, say $\mathscr{I}=\mathscr{I}^{0}$. For $N=2,3$ we obtain 1,3 relations (4.2.8) respectively.

\subsection{The Functional Form of the $A(\chi, m)$}

We distinguish four types of functions:

A. homogeneous polynomials in $s$ of degree $\frac{\omega\left(G_{N}\right)}{2}$,

B. multilinearfunctions of $\ln s_{i} 1 \leqq i \leqq N$ with coefficients of type A

$$
f=\sum_{\chi} c(\chi) \prod_{i \in \chi}\left(\ln s_{i}\right) \quad c(\chi) \in A .
$$

We call $\max |\chi|$ the logarithmic degree of $f$.

C. Polynomials in $s$ of degree $\frac{\omega\left(G_{N}\right)}{2}+1$, divided by $s_{0}$,

D. multilinear functions of $\ln s_{i} 1 \leqq i \leqq N$ with coefficients of type C.

It will follow from the results in this section that $V_{N, \mathscr{I}}$ has an invariant subspace $K$ such that the induced representation of $\mathscr{G}_{N}$ on $V_{N . \mathscr{I}} / K$ is isomorphic with the representation of $\mathscr{G}_{N}$ obtained in the case $m=2(\S 3.3)$.

Lemma 4.3.1. If $|\chi|+|m|=N-1$ and $|m| \geqq 1, A(\chi, m)$ is of type $A$.

Proof. From (4.1.28) we have

$$
b_{i} A(\chi, m)=A(\chi \cup\{i\}, m)=0 \quad 1 \leqq i \leqq N .
$$

Also $a A(\chi, m)=0$ since $A(\chi, m)$ is polynomial in $s_{0}(4.1 .30)$. $A(\chi, m)$ is thus a single-valued function. The only pole of the renormalized amplitude is the second-type singularity $s_{0}=0$ which is a simple pole. But this pole cannot appear in $A(\chi, m)$. To complete the proof of the lemma we must show that $A(\chi, m)$ is homogeneous of degree $\frac{\omega\left(G_{N}\right)}{2}$ in s. Note the renormalized amplitude is not homogeneous in $s$. However, if we write down the equation which expresses the homogeneity of $\mu^{m} \psi_{\eta}$ for generic $\mu$ in the neighbourhood of $\mu=0$ and apply $\mathscr{I}$ to both sides we find that 
$A(\eta, m)$ is homogeneous modulo terms in $A\left(\eta, m^{\prime}\right)$ with $\left|m^{\prime}\right|>|m|$

$$
\begin{gathered}
\mu^{m} \psi_{\eta}(c s)=c^{\omega\left(G_{N}\right) / 2-\Sigma_{2} \mu_{2} / 2 \pi i} \psi_{\eta}(s), \\
A(\eta, m)(c s)=c^{\omega\left(G_{N}\right) / 2} A(\eta, m)(s) \\
+c^{-\Sigma_{\imath} \mu_{l} / 2 \pi i} \sum_{\substack{m^{\prime} \\
1 \leqq\left|m^{\prime}\right| \leqq N-1-|\eta|-|m|}} \frac{(-1)^{\left|m^{\prime}\right|}}{(2 \pi i)^{\left|m^{\prime}\right|}} \frac{(\log c)^{\left|m^{\prime}\right|}}{m^{\prime} !} A\left(\eta, m+m^{\prime}\right)(s) .
\end{gathered}
$$

In the present case $\eta=\chi$ these additional terms vanish so $A(\chi, m)$ is homogeneous.

We remark that (4.3.2) shows that the sheets $A(\eta, m)(s)$ of the renormalized amplitude satisfy

$$
A(\eta, m)(c s)=O\left(|c|^{\omega\left(G_{N}\right) / 2}(\log |c|)^{N-1-|\eta|-|m|}\right) .
$$

Lemma 4.3.4. If $N-1-|\chi|-|m|=j$ and $|m| \geqq 1 A(\chi, m)$ is of type $B$ with logarithmic degree $j$ in the variables $\ln s_{i} i \in \chi^{\prime}$.

The proof is by induction on $j$ starting from the case $j=0$ (Lemma 4.3.1). Consider

$$
B(\chi, m)=\sum_{\psi \subset \chi^{\prime}}(-1)^{|\psi|} \prod_{i \in \psi}\left(\frac{1}{2 \pi i} \ln s_{i}\right) A(\chi \cup \psi, m) .
$$

For $1 \leqq i \leqq N$ we have $b_{i} B(\chi, m)=0$. Since $B(\chi, m)$ is polynomial in $s_{0}$ it follows that it is single valued and nonsingular for all s. By (4.3.3) it is polynomially bounded, and hence a polynomial. By (4.3.2) this polynomial is homogeneous of degree $\omega\left(G_{N}\right) / 2$. By the induction assumption $A(\chi \cup \psi, m)$ is of type B with logarithmic degree $j-|\psi|$ in the variables $\ln s_{k} k \in(\chi \cup \psi)^{\prime}$.

It is interesting to note that if we use (4.3.2) to examine the homogeneity of $B(\chi, m)$ we obtain for $B(\chi, m)(c s)-c^{\omega\left(G_{N}\right) / 2} B(\chi, m)(s)$ a polynomial in $\log c$ multiplied by $c^{\omega\left(G_{N}\right) / 2}$ which must vanish identically in $\log c$. Equating to zero the coefficients of $(\log c)^{t} 1 \leqq t \leqq j$ we obtain a further set of identities on the $A(\chi, m)$.

We now consider the question: do the identities on the vectors $\psi_{x}$ which we established in the case $m=2$ ((3.2.8), (3.3.4), (3.3.6)) persist in the renormalized case? This is answered by Lemma 4.3.6.

Lemma 4.3.6 If $\sum_{\substack{x \in \mathcal{M} \\|\chi|=j}} d_{\chi} \psi_{\chi}=0$ is a linear relation on the vectors $\psi_{\chi} \in V_{N}$ in the case $m=2, N-\left[\frac{N}{2}\right] \leqq j \leqq N-1$

$$
\sum_{\chi \in \mathcal{M},|\chi|=j} d_{\chi} A(\chi, \phi)=D
$$

is a function of type $D$ of logarithmic degree $N-1-j$ in the $\ln s_{i}$.

The proof is by induction on $k=N-1-j$. For $k=0$ we have $b_{i} D=0$ $1 \leqq i \leqq N$. Also $a D=0$ in view of 4.2.1. $D$ is therefore a single-valued 
function in $s$, and can at worst have the $s_{0}=0$ pole. Thus $s_{0} D$ is everywhere nonsingular. From (4.3.2), (4.3.3) it follows that it is a homogeneous polynomial of degree $\omega\left(G_{N}\right)+1$. For $k>0$ we note again that $a D=0$ in view of 4.2.1. Also from the explicit form of the identities in the case $m=2$ we see that for all $\psi|\psi| \geqq 1 b(\psi) D$ is a sum of the same kind with $k^{\prime}<k$ and hence by the induction assumption a function of type $D$ of logarithmic degree $N-1-j-|\psi|$. We consider

$$
B=D+\sum_{\psi:|\psi| \geqq 1}(-1)^{|\psi|} \prod_{i \in \psi}\left(\frac{1}{2 \pi i} \ln s_{i}\right) b(\psi) D .
$$

$b_{i} B=a B=0$ so $B$ is a single-valued function. As in the case $k=0$ it follows that $s_{0} B$ is a homogeneous polynomial of degree $\omega\left(G_{N}\right)+1$.

We remark that 4.3 .6 cannot be improved to give $D$ a polynomial in $s_{0}$. For an explicit calculation in the case $N=2$ gives

$$
A(1,0)-A(2,0)=\frac{s_{1}-s_{2}}{s_{0}} .
$$

\section{References}

1. Plemelj, J.: Problems in the sense of Riemann and Klein. Interscience Tracts in Mathematics 16, 1964.

2. Eden, R. J., P. V. Landshoff, D. I. Olive, and J. C. Polkinghorne: The analytic S-matrix. Cambridge University Press 1966.

3. Hwa, R. C., and V. L. Teplitz: Homology and Feynman integrals. New York: W. A. Benjamin 1966.

4. Pham, F.: Introduction à l'etude topologique des singularitiés de Landau. Paris: Gauthier-Villars 1967

5. Spanter, E. H.: Algebraic topology. McGraw-H1ll 1966.

6. Pham, F.: Ann. Inst. H. Poincaré 6, 89-204 (1967) (Appendix IV).

7. Zarski, O.: Ann. Math. 38, 131-141 (1936).

8. Fotradi, D., M. Froissart, J. Lascoux, and F. Pham: Topology 4, 159-191 (1965) (reprinted in [3]).

9. van Kampen, E. R.: Am. J. Math. 55, 255-260 (1933).

10. Magnus, W., A. Karrass, and D. Solitar: Combinatorial group theory. New York: Interscience $1966(\$ 3.7)$.

11. Ponzano, G., and T. Regge: The monodromy group of one-loop relativistic Feynman integrals. Institute for Advanced Study preprint, Princeton 1968.

12. Lascoux, J.: Perturbation theory in quantum field theory and homology. Battelle Rencontres, 1967, Lectures in Mathematics and Physics, ed. C. M. DeWitt, J. A. Wheeler. New York: W. A. Benjamin 1968.

13. Clemens, C. H.: Picard-Lefschetz theorem for families of algebraıc varieties acquirıng certain singularities. Thesis, Berkeley, 1966.

14. Nilsson, N.: Arkıv Math. 5, 463-476 (1964).

15. Speer, E. R.: J. Math. Phys. 9, 1404 (1968).

G. Ponzano

Istituto di Fisica

University di Torino

Via Giuria 1

I-10125, Torino
T. Regge

E. R. Speer

M. J. Westwater

Institute for Advanced Study

Princeton, N. J. 08540, USA 